CFP-2005-05

$\mathrm{ROM} 2 \mathrm{~F} / 2005 / 10$

\title{
Unitarity in the Presence of Closed Timelike Curves
}

\author{
Lorenzo Cornalba ${ }^{\dagger} \&$ Miguel S. Costa ${ }^{\ddagger}$ \\ †Dipartimento di Fisica \& INFN, Universitá di Roma "Tor Vergata" \\ Via della Ricerca Scientifica 1, 00133, Roma, Italy \\ cornalba@roma2.infn.it \\ $\ddagger$ Departamento de Física e Centro de Física do Porto \\ Faculdade de Ciências da Universidade do Porto \\ Rua do Campo Alegre, 687, 4169-007 Porto, Portugal \\ miguelc@fc.up.pt
}

\begin{abstract}
We conjecture that, in certain cases, quantum dynamics is consistent in the presence of closed timelike curves. We consider time dependent orbifolds of three dimensional Minkowski space describing, in the limit of large AdS radius, BTZ black holes inside the horizon. Although perturbative unitarity fails, we show that, for discrete values of the gravitational coupling, particle propagation is consistent with unitarity. This quantization corresponds to the quantization of the black hole angular momentum, as expected from the dual CFT description. Note, however, that we recover this result by analyzing the physics inside the horizon and near the singularity. The spacetime under consideration has no AdS boundary, and we are therefore not using any assumption regarding a possible dual formulation. We perform the computation at very low energies, where string effects are irrelevant and interactions are dominated by graviton exchange in the eikonal regime. We probe the non-causal structure of space-time to leading order, but work to all orders in the gravitational coupling.
\end{abstract}




\section{Contents}

1 Introduction 1

2 The orbifold 4

2.1 Geometrv . . . . . . . . . . . . . . . . . 6

2.2 Particle wavefunctions . . . . . . . . . . . . 8

3 Fevnman rules 11

4 Propagation in the presence of CCC's $\quad 15$

4.1 One-loop tadpole . . . . . . . . . . . . . . . . . . . 18

4.2 Two-point function . . . . . . . . . . . . . . . . . . 20

4.3 Eikonal approximation . . . . . . . . . . . . . . . 24

4.4 Quantization condition from unitarity . . . . . . . . . . 28

5 Extending the results to off-shell external states $\quad 31$

5.1 Particle states . . . . . . . . . . . . . . . . . . . 32

6 Conclusions 34

\section{Introduction}

One of the outstanding difficulties of the AdS/CFT correspondence [1] is to understand physics in the bulk of the AdS space in terms of CFT data. In particular, understanding the space-time causal structure of black holes is still a fundamental problem from the view point of the duality. Although one believes, based on basic properties of the dual CFT, that the bulk dynamics is well defined, it is fair to say that the quantum nature of horizons and singularities remains rather mysterious [2].

The $\mathrm{AdS}_{3} / \mathrm{CFT}_{2}$ case is one of the best studied examples of the duality, with black hole geometries given by the BTZ metric [3, 4]

$$
d s^{2}=-N^{2} d t^{2}+N^{-2} d r^{2}+r^{2}\left(d \phi-N_{\phi} d t\right)^{2},
$$

where

$$
N^{2}=\frac{1}{\ell^{2} r^{2}}\left(r^{2}-r_{+}^{2}\right)\left(r^{2}-r_{-}^{2}\right), \quad \quad N_{\phi}=\frac{1}{\ell} \frac{r_{+} r_{-}}{r^{2}} .
$$

The $\mathrm{AdS}_{3}$ radius is given by $\ell$, and $r_{+}, r_{-}$are the positions of the outer and inner horizons determining the mass and the angular momentum of the 
black hole

$$
M_{\mathrm{bh}}=\frac{\pi M}{4}\left(\frac{r_{+}^{2}+r_{-}^{2}}{\ell^{2}}+1\right), \quad J=\frac{\pi M}{2} \frac{r_{+} r_{-}}{\ell},
$$

in terms of the three-dimensional Planck mass ${ }^{1} M$.

In the dual $\mathrm{CFT}_{2}$ description, these black holes correspond to states with [5]

$$
L_{0}+\tilde{L}_{0}=\ell M_{\mathrm{bh}}, \quad L_{0}-\tilde{L}_{0}=J
$$

where $L_{0}, \tilde{L}_{0}$ are the Virasoro zero modes. Moreover, since the $\phi$ circle is a non-contractible loop in spacetime, in the presence of fermions one needs to choose a spin structure. Usually one considers periodic boundary conditions for the fermions, which allow for a covariantly constant spinor in the extremal case $r_{+}=r_{-}$and which give a state in the Ramond sector of the $\mathrm{CFT}_{2}$. One may also choose antiperiodic boundary conditions, which describe a non-supersymmetric state in the NS sector of the $\mathrm{CFT}_{2}$. From this point of view, the spin eigenvalue $J$ is naturally quantized in half integral units

$$
2 J \in \mathbb{Z} .
$$

On the other hand, from a purely gravitational view point, the quantization of the angular momentum is rather mysterious. Classically, $J$ is a continuous parameter, and the usual arguments leading to (2) rely on the asymptotic symmetries of quantum gravity on $\mathrm{AdS}_{3}[6]$, and therefore implicitly on the existence of a dual $\mathrm{CFT}_{2}$.

An intriguing property of the BTZ black holes is the existence of closed causal curves (CCC's) in the geometry. In fact, these holes are quotients of $\mathrm{AdS}_{3}$ by the action of a specific isometry parameterized by $r_{+}, r_{-}$, and the identification creates CCC's located in the region inside the inner horizon. The black hole has a chronological singularity where the generator of the orbifold isometry becomes null. Therefore, if we ignore the dual CFT description, we naively expect that quantum gravity in the BTZ geometry presents pathologies due to the existence of these CCC's. In particular, one would a priori expect violations of unitarity, which would undermine the possible existence of an $S$ matrix.

By studying quantum field theory in the flat space limit $\ell \rightarrow \infty$ of the BTZ geometry, we shall show that the quantization condition (2) can be

\footnotetext{
${ }^{1}$ For notational convenience, we normalize the Planck mass in terms of the Newton constant $G$ as $M^{-1}=2 \pi G$.
} 


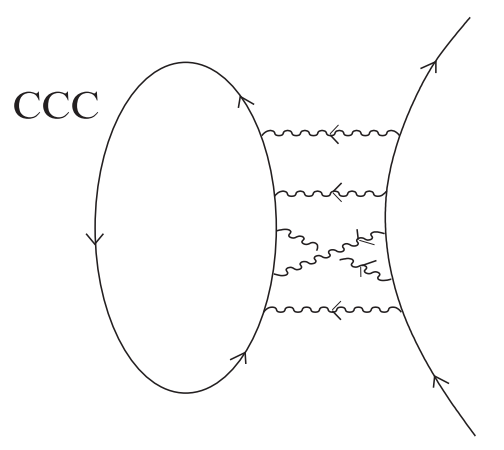

Figure 1: Leading correction to the free propagation of a scalar field due to gravitational interactions with virtual particles winding closed causal curves.

obtained by demanding that quantum propagation of fields is consistent with unitarity, even in the presence of CCC's. The argument will use very limited information about the underlying quantum gravity. In particular, we shall not use any string theoretic arguments, since we shall work at energies well below the string scale, where $\alpha^{\prime}$ corrections should be negligible. More specifically, we will consider corrections to free propagation of scalar fields due to interactions with particles winding around the closed timelike direction, as shown in Figure 1. By carefully choosing the quantum numbers of the external states, we will show, using general arguments [7, that the interaction is dominated by graviton exchange in the eikonal regime. In this kinematical regime, one has enough control over the resummation of the perturbative series determining the gravitational interaction, and one can recover the quantization condition (2) by enforcing unitarity.

For a given BTZ black hole with geometric parameters $r_{+}, r_{-}$, one could in principle start by considering quantum gravity at arbitrary values of the gravitational coupling $M^{-1}$. However, the presence of CCC's breaks unitarity order by order in the coupling constant [8], suggesting that one is not free to choose $M^{-1}$ at will and that one needs to resum the perturbation series. The quantization condition (2), which follows from unitarity, can then be interpreted as fixing the gravitational coupling $M^{-1}$ at some specific values.

The usual belief regarding the dynamics inside the horizons of a BTZ black hole is that the geometry (11) will be modified due to the classical instability of the inner horizon [9] and also due to divergences of the quantum stress-energy tensor at polarization surfaces in the region inside the inner horizon [10]. We propose, on the other hand, that the geometry remains unaltered and non-causal, but the propagation of states is strongly modified 
by quantum effects in the inner region and is consistent whenever (2) is satisfied. It is tempting to speculate that such modification of the dynamics inside the horizons is responsible for the reduction of degrees of freedom associated to the black hole entropy, which is not proportional to the volume of the black hole but to its horizon area.

\section{The orbifold}

For simplicity we focus on the extremal black hole, with $r_{+}=r_{-}$and Penrose diagram given in Figure 2 $\mathrm{a}$, which is a quotient of $\mathrm{AdS}_{3}$ space. In the flat space $\ell \rightarrow \infty$ limit, keeping the energy scale

$$
E=\frac{\ell}{\left(2 \pi r_{+}\right)^{2}}
$$

fixed, the region inside the black hole horizon becomes an orbifold of flat Minkowski space $\mathbb{M}^{3} / e^{\kappa}$, introduced in [1]. Choosing coordinates $x^{ \pm}, x$ on $\mathbb{M}^{3}$, such that the metric is

$$
d s^{2}=-2 d x^{+} d x^{-}+d x^{2},
$$

the orbifold generator $\kappa$ is the Killing vector

$$
\kappa=i\left(L_{+x}+E^{-1} K_{-}\right)=-\left(x^{-} \partial_{x}+x \partial_{+}\right)+E^{-1} \partial_{-},
$$

where $L_{a b}, K_{a}$ are, respectively, the generators of Lorentz transformations and translations, and where $E$ parameterizes inequivalent orbifolds. For a detailed derivation of the $\ell \rightarrow \infty$ limiting procedure, we refer the reader to appendix A.

Under the change of coordinates

$$
\begin{aligned}
x^{+} & =y^{+}-E y y^{-}+\frac{E^{2}}{6}\left(y^{-}\right)^{3}, \\
x^{-} & =y^{-}, \\
x & =y-\frac{E}{2}\left(y^{-}\right)^{2},
\end{aligned}
$$

the metric becomes

$$
d s^{2}=-2 d y^{+} d y^{-}+2 E y\left(d y^{-}\right)^{2}+d y^{2}
$$

and the Killing vector

$$
\kappa=\frac{1}{E} \frac{\partial}{\partial y^{-}} .
$$




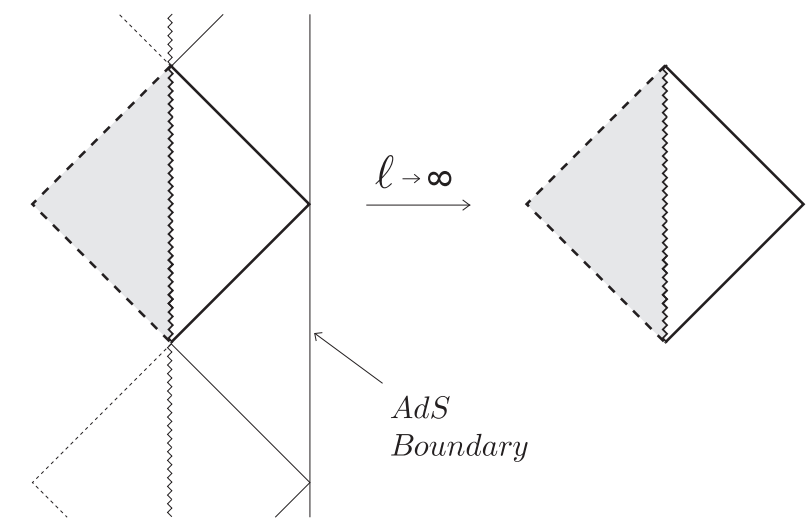

(a)

(b)

Figure 2: Penrose diagram of the extremal BTZ black hole (a). The shaded area represents the region behind the chronological singularity, where closed causal curves are present. In the limit $\ell \rightarrow \infty, J$ fixed, one focuses on the region inside the black hole horizon and obtains a flat space orbifold with Penrose diagram (b).

The direction $y^{-}$is therefore compact with period

$$
y^{-} \sim y^{-}+\frac{1}{E} .
$$

Note that the form of the metric (4) can be directly obtained from the BTZ metric (11) for $r_{+}=r_{-}$, by setting $y=2 \pi^{2} E r^{2}, 2 \pi y^{+}=t, 2 \pi E y^{-}=\phi$, and then by taking the limit $\ell \rightarrow \infty$ described above. Re-expressing the metric (41) as

$$
d s^{2}=-\frac{\left(d y^{+}\right)^{2}}{2 E y}+d y^{2}+2 E y\left(d y^{-}-\frac{d y^{+}}{2 E y}\right)^{2},
$$

we can easily draw the corresponding Penrose diagram as in Figure $2 \mathbf{b}$, showing that we are focusing on the region inside the horizon of the extremal BTZ black hole. In the non-extremal case a similar large $\ell$ limit leads to the shift-boost orbifold of $\mathbb{M}^{3}$, which focuses on the region inside the outer horizon [12].

The quantization of the BTZ black hole angular momentum (2) becomes, in the flat space limit, the condition

$$
2 J=\frac{M}{4 \pi E} \in \mathbb{Z} .
$$

In this case, on the other hand, one cannot justify this quantization condition with arguments relying on asymptotic symmetries and on the existence of a dual CFT. In fact, the Minkowski space orbifold just described focuses 
on the region inside the horizons, and the asymptotic AdS boundary is no longer part of the geometry.

An independent way of deriving (5) is to embed the orbifold in string theory, by considering Type II strings on $\mathbb{M}^{3} / e^{\kappa} \times \mathbb{T}^{7}$ [11]. After a sequence of dualities, the geometry becomes that of an orientifold $O 8$ plane [13. From this point of view, (5) results from the fact that 8-dimensional RR charged objects have charges quantized in units of the $D 8$-brane charge. Although these arguments rely on the low-energy supergravity description of the system, they lead to the same condition.

It is the main point of this paper to derive the quantization condition (5) purely within the framework of quantum field theory in the presence of gravitational interactions. From this perspective, (5) is obtained by requiring unitarity in the space $\mathbb{M}^{3} / e^{\kappa}$, which possess CCC's. Hence we see that unitarity in the presence of CCC's is related to charge quantization in dual descriptions of the system. The mechanics that protects chronology is rather different than that proposed by Hawking [14, which is based on a large backreaction due to UV effects.

For other studies of orbifolds with CCC's, where an analogous study of unitarity can in principle be done, see [15-32].

\subsection{Geometry}

We now analyze, in more detail, the geometry of the orbifold $\mathbb{M}^{3} / e^{\kappa}$. First let us note that the square-norm of $\kappa$ is given by

$$
\kappa^{2}=\frac{2 y}{E} .
$$

The compact $y^{-}$circle is space-like for $y>0$ and timelike for $y<0$. Therefore, the geometry has CCC's. It is simple to show that all CCC's must go in the region with $y<0$. To prove this fact, assume that we have a CCC parameterized by $y^{a}(\lambda)$ with $\lambda \in[0,1]$. Since $y^{+}(0)=y^{+}(1)$, the function $y^{+}(\lambda)$ must achieve an extremum for some $\bar{\lambda} \in(0,1)$. At $\lambda=\bar{\lambda}$, the metric (4) reduces to $2 E y\left(d y^{-}\right)^{2}+d y^{2}$, which is positive-definite for $y>0$. Therefore, the curve can be timelike or null only if $y(\bar{\lambda}) \leq 0$. A schematic representation of these basic features of the geometry is shown in Figure 3 .

A particularly interesting class of closed curves are the ones obtained from geodesics in the covering space $\mathbb{M}^{3}$ connecting points which are related 


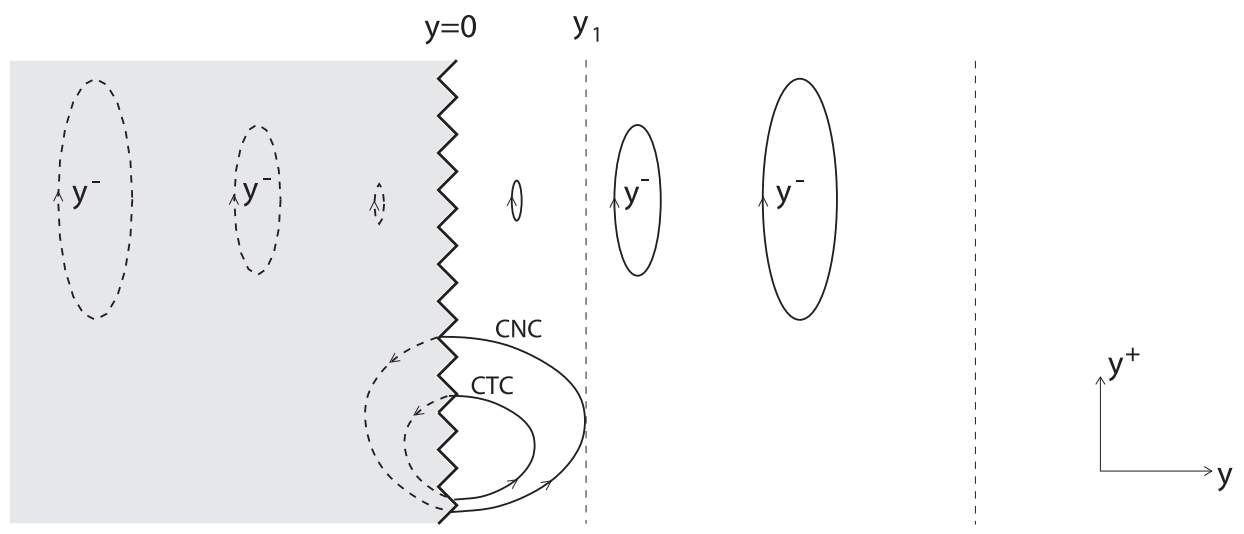

Figure 3: A representation of the basic features of the orbifold geometry. The null Killing direction $y^{+}$and the transverse direction $y$ are drawn on the plane. The compact $y^{-}$circle is spacelike for positive $y$ and timelike in the shaded region after the chronological singularity. Represented are also the first polarization surfaces at $y_{w}=w^{2} /(24 E)$. Finally we show a closed timelike curve and a closed null curve. The latter starts and ends on the first polarization surface.

by the action of the orbifold group. Denoting, for notational convenience, the orbifold group generator by

$$
\Omega=e^{\kappa},
$$

it is a simple matter to show, starting from (3), that the continuous transformation $y^{-} \rightarrow y^{-}+s / E$ generated by $\Omega^{s}$ reads, in the coordinates $x^{a}$,

$$
\begin{aligned}
\left(\Omega^{s} \mathbf{x}\right)^{+} & =x^{+}-s x+\frac{s^{2}}{2} x^{-}+\frac{s^{3}}{6 E} \\
\left(\Omega^{s} \mathbf{x}\right)^{-} & =x^{-}+\frac{s}{E} \\
\left(\Omega^{s} \mathbf{x}\right) & =x-s x^{-}-\frac{s^{2}}{2 E} .
\end{aligned}
$$

Therefore, the geodesic distance squared $\left(\Omega^{w} \mathbf{x}-\mathbf{x}\right)^{2}$ between a point $\mathbf{x}$ and its $w$-th image $(w \in \mathbb{Z})$ is given by

$$
\frac{2 w^{2}}{E}\left(y-\frac{w^{2}}{24 E}\right)
$$

and becomes null at the so-called polarization surfaces

$$
y=\frac{w^{2}}{24 E} \text {. }
$$

For $y<w^{2} /(24 E)$ the geodesic from $\mathbf{x}$ to $\Omega^{w} \mathbf{x}$ is a closed timelike curve. The polarization surfaces are also represented in Figure 3 , 


\subsection{Particle wavefunctions}

In order to analyze the propagation of scalar fields in the orbifold geometry, we must first find a convenient basis of functions invariant under the orbifold action. It is useful to consider first the transformation of the plane waves

$$
\phi_{\mathbf{k}}(\mathbf{x})=e^{i \mathbf{k} \cdot \mathbf{x}}
$$

under the action of $\Omega$. Using (6), it is simple to show that

$$
\phi_{\mathbf{k}}\left(\Omega^{-s} \mathbf{x}\right)=\phi_{\Omega^{s} \mathbf{k}}(\mathbf{x}) e^{-i \varphi(\mathbf{k}, s)},
$$

where the transformed momentum $\Omega^{s} \mathbf{k}$ is given by

$$
\begin{aligned}
\left(\Omega^{s} \mathbf{k}\right)_{+} & =k_{+}, \\
\left(\Omega^{s} \mathbf{k}\right)_{-} & =k_{-}+s k+\frac{s^{2}}{2} k_{+}, \\
\left(\Omega^{s} \mathbf{k}\right) & =k+s k_{+} .
\end{aligned}
$$

The above phase $\varphi(\mathbf{k}, s)$ is given by

$$
\varphi(\mathbf{k}, s)=\frac{1}{E}\left(\frac{s^{3}}{6} k_{+}+s k_{-}+\frac{s^{2}}{2} k\right),
$$

and satisfies $\varphi(\mathbf{k}, s+t)=\varphi(\mathbf{k}, s)+\varphi\left(\Omega^{s} \mathbf{k}, t\right)$.

We can construct, starting from any plane wave $\phi_{\mathbf{k}}(\mathbf{x})$, a function on $\mathbb{M}^{3}$ which is invariant under $\Omega$ and which is an eigenvector of $\kappa$ with eigenvalue $2 \pi i n$, where $n \in \mathbb{Z}$. This is given by the integral representation

$$
\int d s e^{2 \pi i n s} \phi_{\mathbf{k}}\left(\Omega^{-s} \mathbf{x}\right)=\int d s \phi_{\Omega^{s} \mathbf{k}}(\mathbf{x}) e^{2 \pi i n s-i \varphi(\mathbf{k}, s)} .
$$

Since $\left(\Omega^{s} \mathbf{k}\right)^{2}=\mathbf{k}^{2}$, and since $\left(\Omega^{s} \mathbf{k}\right)_{+}=k_{+}$, the above functions are automatically eigenvectors of the Laplacian $\square$ and of the momentum operator $K_{+}$. We then choose, as a convenient basis for the invariant functions on $\mathbb{M}^{3} / e^{\kappa}$, eigenfunctions $V_{\lambda, p_{+}, p_{-}}(\mathbf{x})$ of the commuting operators $\square, K_{+}$and $\kappa$, with eigenvalues

$$
\begin{aligned}
\square & =\lambda, \\
K_{+} & =p_{+}, \\
-i E \kappa & =p_{-},
\end{aligned}
$$


where $p_{-}$is related to the eigenvalue $n$ by $^{2}$

$$
p_{-}=2 \pi E n .
$$

Choosing, in (9), the momentum $\mathbf{k}=\left(p_{+}, \lambda / 2 p_{+}, 0\right)$, we immediately obtain the explicit representation

$$
\begin{aligned}
V_{\lambda, p_{+}, p_{-}}(\mathbf{x})= & \frac{1}{\left|p_{+}\right|} \int d k e^{i\left(p_{+} x^{+}+k_{-} x^{-}+k x\right)} \times \\
& \times \exp \frac{i}{2 E p_{+}^{2}}\left[\left(2 p_{+} p_{-}-\lambda\right) k-\frac{k^{3}}{3}\right],
\end{aligned}
$$

where we have changed the integration variable to $k=s p_{+}$, and where $k_{-}$ is given by the on-shell condition

$$
k_{-}=\frac{k^{2}+\lambda}{2 p_{+}} .
$$

The sector with $p_{+}=0$ clearly requires a separate treatment. In this case, (8) implies that the component $k=\left(\Omega^{s} \mathbf{k}\right)$ is invariant under $\Omega$, and we can choose eigenfunctions $V_{p, p_{-}}(\mathbf{x})$ with

$$
\begin{aligned}
K & =p, \\
-i E \kappa & =p_{-},
\end{aligned}
$$

where $K$ is the momentum operator $-i \partial_{x}$. Choosing, in (9), the momentum $\mathbf{k}=(0,0, p)$, and as integration variable $k_{-}=s p$, we obtain the integral representation

$$
V_{p, p_{-}}(\mathbf{x})=\frac{1}{|p|} \int d k_{-} e^{i\left(k_{-} x^{-}+p x\right)} \exp \frac{i}{p E}\left[p_{-} k_{-}-\frac{\left(k_{-}\right)^{2}}{2}\right],
$$

which has $p_{+}=0$ and $\lambda=-p^{2}$.

The functions $V_{\lambda, p_{+}, p_{-}}$and $V_{p, p_{-}}$represent a complete basis of invariant functions on $\mathbb{M}^{3} / e^{\kappa}$, and transform, under conjugation, as

$$
\begin{aligned}
V_{\lambda, p_{+}, p_{-}}^{\star}(\mathbf{x}) & =V_{\lambda,-p_{+},-p_{-}}(\mathbf{x}), \\
V_{p_{,} p_{-}}^{\star}(\mathbf{x}) & =V_{-p,-p_{-}}(\mathbf{x}) .
\end{aligned}
$$

\footnotetext{
${ }^{2}$ Throughout the paper, we use $\mathbf{k}$, $\mathbf{q}$ to denote covering space momenta $-i$.e eigenvalues of $-i \partial_{\mathbf{x}}$. We reserve $p_{ \pm}$for the momenta in the $\mathbf{y}$ coordinates $-i \partial_{y^{ \pm}}$
} 
It is useful to consider the above functions in the coordinates $y^{ \pm}, y$. The operators in (10) are given, in these coordinates, by

$$
\begin{aligned}
\square & =-2 \partial_{y^{+}} \partial_{y^{-}}-2 E y \partial_{y^{+}}^{2}+\partial_{y}^{2}, \\
K_{+} & =-i \partial_{y^{+}}, \\
-i E \kappa & =-i \partial_{y^{-}} .
\end{aligned}
$$

We can then use separation of variables to find functions satisfying (10) of the form $f(y) e^{i\left(p_{+} y^{+}+p_{-} y^{-}\right)}$, where $f(y)$ solves

$$
\left(2 p_{+} p_{-}+2 E p_{+}^{2} y+\frac{d^{2}}{d y^{2}}-\lambda\right) f(y)=0 .
$$

Defining the dimensionless variable

$$
z=\left(2 E p_{+}^{2}\right)^{\frac{1}{3}}\left(y-y_{0}\right), \quad\left(y_{0}=\frac{\lambda-2 p_{+} p_{-}}{2 E p_{+}^{2}}\right)
$$

the above differential equation simplifies to $d^{2} f / d z^{2}+z f=0$, which describes in quantum mechanics a zero energy particle subject to a linear potential. The solutions are the Airy functions $\mathrm{Ai}(-z)$ and $\mathrm{Bi}(-z)$, which are, respectively, exponentially damped and exponentially growing in the $z<0$ region. This region corresponds mostly to negative $y$, where the Killing vector $\kappa$ is time-like. In the sequel, we shall consider the normalizable solution $\mathrm{Ai}(-z)$ which corresponds to the integral representation (11). Using the representation of the Airy function

$$
\operatorname{Ai}(-z)=\frac{1}{2 \pi} \int d t e^{i\left(z t-\frac{t^{3}}{3}\right)}
$$

it is a matter of computation to show, using (3) and (11), that

$$
V_{\lambda, p_{+}, p_{-}}(\mathbf{y})=2 \pi\left(\frac{2 E}{\left|p_{+}\right|}\right)^{\frac{1}{3}} \operatorname{Ai}(-z) e^{i\left(p_{+} y^{+}+p_{-} y^{-}\right)} .
$$

The case $p_{+}=0$ is simpler since in the differential equation (13) the linear potential term is absent. The solutions are simply plane waves $f(y) \propto e^{ \pm i p y}$, with $p^{2}=-\lambda$. In fact, we can explicitly integrate (12) and obtain

$$
V_{p, p_{-}}(\mathbf{y})=\sqrt{\frac{2 \pi E}{i p}} e^{i\left(p y+p_{-} y^{-}\right)} .
$$


Finally, the inner product of the wave functions is given by

$$
\begin{aligned}
\int_{\mathcal{F}} d^{3} \mathbf{x} V_{\lambda, p_{+}, p_{-}}(\mathbf{x}) V_{\lambda^{\prime}, p_{+}^{\prime}, p_{-}^{\prime}}^{\star}(\mathbf{x}) & =16 \pi^{3} \delta\left(\lambda-\lambda^{\prime}\right) \delta\left(p_{+}-p_{+}^{\prime}\right) \delta_{p_{-}-p_{-}^{\prime}}, \\
\int_{\mathcal{F}} d^{3} \mathbf{x} V_{p, p_{-}}(\mathbf{x}) V_{p^{\prime}, p_{-}^{\prime}}^{\star}(\mathbf{x}) & =\frac{4 \pi^{2}}{|p|} T \delta\left(p-p^{\prime}\right) \delta_{p_{-}-p_{-}^{\prime}}
\end{aligned}
$$

where $T=\int d y^{+}=2 \pi \delta\left(p_{+}=0\right)$ is the volume in the $y^{+}$direction and $\mathcal{F} \subset \mathbb{M}^{3}$ is a fundamental domain of the orbifold (we can consider $\mathcal{F}$ to be, for concreteness, the region $0<y^{-}<1 / E$ ). A simple way to prove the above expressions is to use the integral representations (11) and (12) and to extend the integration region from $\mathcal{F}$ to $\mathbb{M}^{3}$. One then uses the orthogonality of the plane waves $\phi_{\mathbf{k}}(\mathbf{x})$ to derive the desired result. The overcounting due to the extension of the integration region can be taken into account by substituting, at the end of the computation, the Dirac $\delta-$ function with the Kronecher symbol for the discrete Kałuża-Klein charge as follows

$$
\begin{aligned}
\delta\left(n-n^{\prime}\right) & \rightarrow \delta_{n-n^{\prime}}, \\
2 \pi \delta\left(p_{-}-p_{-}^{\prime}\right) & \rightarrow E^{-1} \delta_{p_{-}-p_{-}^{\prime}} .
\end{aligned}
$$

The correctness of the normalization in the above expression can be checked, for instance, by computing (15) directly in the $y$-coordinates, using the explicit expression (14).

\section{$3 \quad$ Feynman rules}

We shall start our investigation of quantum field theory in the orbifold space by considering, as a simple toy model, a scalar field $\Phi$ with a cubic coupling, described by the action

$$
\int_{\mathcal{F}} d^{3} \mathbf{x}\left[\frac{1}{2} \Phi\left(\square-m^{2}\right) \Phi+\frac{g}{6} \Phi^{3}\right] .
$$

We shall analyze the perturbative expansion of this theory in some detail, since some of the basic features of field and string theory on $\mathbb{M}^{3} / e^{\kappa}$ can be already understood in this simple setting. Later on we will be interested in the free theory of a scalar field coupled to gravity.

Consider first the scalar propagator, which is simply given by the method of images. Denoting the Feynman propagator in the covering space by

$$
\Delta\left(\mathbf{x}, \mathbf{x}^{\prime}\right)=\int \frac{d^{3} \mathbf{q}}{(2 \pi)^{3}} \frac{-i}{\mathbf{q}^{2}+m^{2}-i \epsilon} e^{i \mathbf{q}\left(\mathbf{x}-\mathbf{x}^{\prime}\right)},
$$




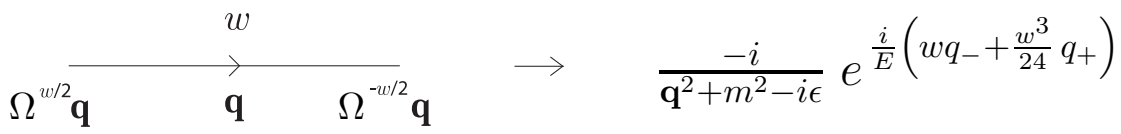

Figure 4: Scalar propagator for a particle winding $w$ times the compact $y^{-}$direction. The incoming and outgoing momenta are related by the action of the orbifold generator and the usual propagator is multiplied by a momentum dependent phase.

we can write the full propagator as a sum

$$
\left\langle\Phi(\mathbf{x}) \Phi\left(\mathbf{x}^{\prime}\right)\right\rangle=\sum_{w \in \mathbb{Z}} \Delta\left(\Omega^{w} \mathbf{x}, \mathbf{x}^{\prime}\right) .
$$

Lorentz invariance implies that $\Delta\left(\Omega^{s} \mathbf{x}, \Omega^{s} \mathbf{x}^{\prime}\right)=\Delta\left(\mathbf{x}, \mathbf{x}^{\prime}\right)$, and therefore we can write the summand $\Delta\left(\Omega^{w} \mathbf{x}, \mathbf{x}^{\prime}\right)=\Delta\left(\Omega^{w / 2} \mathbf{x}, \Omega^{-w / 2} \mathbf{x}^{\prime}\right)$ symmetrically as

$$
\begin{aligned}
& \int \frac{d^{3} \mathbf{q}}{(2 \pi)^{3}} \frac{-i}{\mathbf{q}^{2}+m^{2}-i \epsilon} \phi_{\mathbf{q}}\left(\Omega^{w / 2} \mathbf{x}\right) \phi_{\mathbf{q}}^{\star}\left(\Omega^{-w / 2} \mathbf{x}^{\prime}\right) \\
& \quad=\int \frac{d^{3} \mathbf{q}}{(2 \pi)^{3}} \frac{-i}{\mathbf{q}^{2}+m^{2}-i \epsilon} e^{\frac{i}{E}\left(w q_{-}+\frac{w^{3}}{24} q_{+}\right)} \phi_{\Omega^{-w / 2} \mathbf{q}}(\mathbf{x}) \phi_{\Omega^{w / 2} \mathbf{q}}^{\star}\left(\mathbf{x}^{\prime}\right) .
\end{aligned}
$$

In the last equation we have used equation (7) to obtain the phase

$$
\varphi\left(\mathbf{q}, \frac{w}{2}\right)-\varphi\left(\mathbf{q},-\frac{w}{2}\right) .
$$

¿From equation (18) we deduce that, in Fourier space, a scalar propagator is labeled by a momentum $\mathbf{q}$ and a winding number $w$. The propagator is then given by

$$
\frac{-i}{\mathbf{q}^{2}+m^{2}-i \epsilon} e^{\frac{i}{E}\left(w q_{-}+\frac{w^{3}}{24} q_{+}\right)} .
$$

Moreover, as we move along the propagator, the momentum gets transformed under the action of the orbifold group element $\Omega^{-w}$. Therefore, the incoming momentum along the line is $\Omega^{w / 2} \mathbf{q}$ and the outgoing one is $\Omega^{-w / 2} \mathbf{q}$, as shown in Figure 4. We have made an explicit choice of $i \epsilon$ prescription for the propagator, which is implicitly a choice of vacuum for the spacetime under consideration. This choice is canonical in orbifold theories, and for BTZ black holes corresponds to the usual Hartle-Hawking vacuum.

We are now ready to state the Feynman rules for computing the amputated $n$-point amplitude of a given connected graph $G$. We are not going to give a formal proof of these rules, since it is simple but notationally quite 


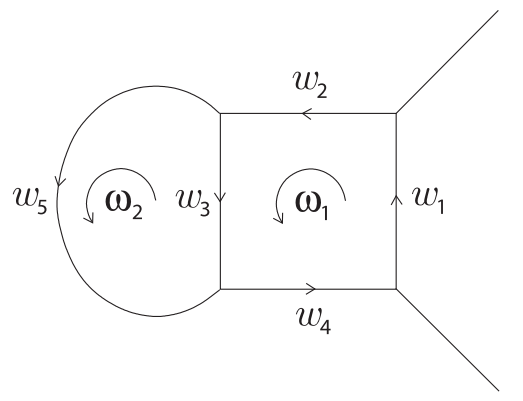

Figure 5: A two-loop graph, with loop winding numbers $\omega_{1}, \omega_{2}$. The winding numbers $w_{i}$ for the propagators can be chosen arbitrarily, as long as $\omega_{1}=\sum_{i=1}^{4} w_{i}$ and $\omega_{2}=w_{5}-w_{3}$.

cumbersome. We hope that the reader with some familiarity with the standard techniques of field theory can convince (her)himself of the validity of the rules below.

i) First assign winding numbers $w_{i}$ to all internal propagators of $G$. This defines a 1-cocycle for the graph, and therefore an element of the cohomology group $\omega \in H^{1}(G)$. We can think of propagators in the graph literally as particle propagation. The numbers $w_{i}$ define how many times the particle winds around the compact $y^{-}$circle as one goes around a loop of $G$. For the element $\omega \in H^{1}(G)$, the intermediate results of the computation will depend on the specific choice of representative $w_{i}(\omega)$, but the final result will only depend on the class $\omega$. We should consider as distinct only choices of windings $w_{i}$ corresponding to different classes in $H^{1}(G)$. As an illustration, Figure 5 shows the choices of winding numbers and classes for a given two-loop graph.

ii) Fix external momenta $\mathbf{k}_{i}$ flowing into the graph, and compute the diagram with the usual Feynman rules, but with propagators given by the above prescription. The result will depend on the explicit choice of representative $w_{i}(\omega)$. Let us denote it by

$$
\Gamma_{w_{i}}\left(\mathbf{k}_{1}, \cdots, \mathbf{k}_{n}\right)
$$

The momenta $\mathbf{k}_{i}$ should be thought of as covering space momenta, even though $\Gamma_{w_{i}}$ is not in general Lorentz invariant. However, since 
invariance under $K_{+}$is preserved, the above amplitude will always contain a delta function $2 \pi \delta\left(\Sigma_{i} k_{i+}\right)$.

iii) Average over the action of the group on the external states as we did for the particle wave functions, by considering the integral

$$
\int d s_{1} \cdots d s_{n} e^{i \chi\left(s_{i}\right)} \Gamma_{w_{i}}\left(\Omega^{s_{1}} \mathbf{k}_{1}, \cdots, \Omega^{s_{n}} \mathbf{k}_{n}\right)
$$

with

$$
\chi\left(s_{i}\right)=2 \pi \sum_{i} s_{i} n_{i}-\sum_{i} \varphi\left(\mathbf{k}_{i}, s_{i}\right) .
$$

The above integral overcounts the result, since invariance under the orbifold action $\kappa$ implies that

$$
e^{i \chi\left(s_{i}\right)} \Gamma_{w_{i}}\left(\Omega^{s_{i}} \mathbf{k}_{i}\right)=e^{2 \pi i s \sum_{i} n_{i}} e^{i \chi\left(s_{i}^{\prime}\right)} \Gamma_{w_{i}}\left(\Omega^{s_{i}^{\prime}} \mathbf{k}_{i}\right),
$$

where $s_{i}=s_{i}^{\prime}+s$. We may insert, in (19), the identity "1"

$$
\left|\sum_{i} c_{i}\right| \int d s \delta\left(\sum_{i} c_{i} s_{i}^{\prime}\right)
$$

where the constants $c_{i}$ can be chosen arbitrarily, as long as $\sum_{i} c_{i} \neq 0$. Changing integration variables to the $s_{i}^{\prime}$, we can perform the integral over $s$ and obtain a delta function $\delta\left(\sum_{i} n_{i}\right)$. Restricting the integral over $s$ to a single action of the orbifold generator, from 0 to 1 , or following the prescription in (16), we can substitute this $\delta$-function with a Kronecher symbol. Dropping the primes we arrive at the amplitude

$$
\delta_{\Sigma n_{i}}\left|\sum_{i} c_{i}\right| \int d s_{1} \cdots d s_{n} \delta\left(\sum_{i} c_{i} s_{i}\right) e^{i \chi\left(s_{i}\right)} \Gamma_{w_{i}}\left(\Omega^{s_{i}} \mathbf{k}_{i}\right)
$$

This expression depends only on the specific class $\omega \in H^{1}(G)$ and it is the final result given a specific choice of $\omega$ and of external states.

iv) Finally sum over $\omega$. The term with $\omega=0$ is singled out since it comes from the parent theory in $\mathbb{M}^{3}$. In fact, we can choose $w_{i}=0$ for all internal propagators and, in this case, $\Gamma_{w_{i}}\left(\mathbf{k}_{i}\right)$ can be computed with the usual flat space Feynman propagator. The only sign of the orbifold would then be in the choice of external states. If $G$ is a treelevel graph, then $H^{1}(G)=0$ and the only term in the sum comes from the parent theory. This is usually called the inheritance principle. 
To do computations, it is convenient to specialize the formula (20) to the external states given by the functions $V_{\lambda, p_{+}, p_{-}}$and $V_{p, p_{-}}$. Consider first the case when all $n$ external states have $p_{+} \neq 0$ and are labeled by $\lambda_{i}, p_{i+}$, $p_{i-}$. We must then compute the following integral (rescaling $c_{i} \rightarrow c_{i} p_{i+}$ )

$$
\delta_{\Sigma p_{i-}}\left|\frac{\sum_{i} c_{i} p_{i+}}{\prod_{i} p_{i+}}\right| \int d k_{1} \cdots d k_{n} \delta\left(\sum_{i} c_{i} k_{i}\right) e^{i \chi\left(k_{i}\right)} \Gamma_{w_{i}}\left(\mathbf{k}_{i}\right),
$$

where the phase $\chi$ is given by

$$
\chi\left(k_{i}\right)=\sum_{i} \frac{1}{2 E p_{i+}^{2}}\left[\left(2 p_{i+} p_{i-}-\lambda_{i}\right) k_{i}-\frac{k_{i}^{3}}{3}\right],
$$

and where

$$
k_{i+}=p_{i+}, \quad k_{i-}=\frac{\lambda_{i}+k_{i}^{2}}{2 p_{i+}} .
$$

If, on the other hand, we have $n+1$ external states, one of which has $p_{+}=0$ and therefore is labeled by $p, p_{-}$, we may choose the corresponding variable $s$ to vanish in (20) and obtain

$$
\delta_{p_{-}+\Sigma p_{i-}}\left|\frac{1}{\prod_{i} p_{i+}}\right| \int d k_{1} \cdots d k_{n} e^{i \chi\left(k_{i}\right)} \Gamma_{w_{i}}\left(\mathbf{k}, \mathbf{k}_{i}\right)
$$

where $\mathbf{k}=(0,0, p)$ and $i$ runs over the remaining $n$ external states.

\section{Propagation in the presence of CCC's}

We have now the basic tools to address the main issue of this paper, namely the analysis of particle propagation in the presence of CCC's. We shall

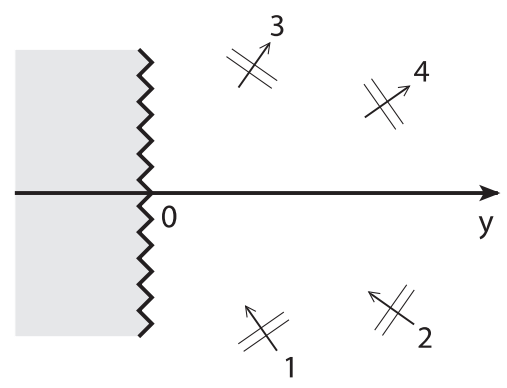

Figure 6: Scattering process in the orbifold geometry. The dynamics is strongly coupled in the shaded region due to gravitational interactions in the presence of CCC's, which naively violate unitarity. We expect, on the other hand, to be able to define particle states and a consistent S-matrix for discrete values of the gravitational coupling constant $M^{-1}$. 


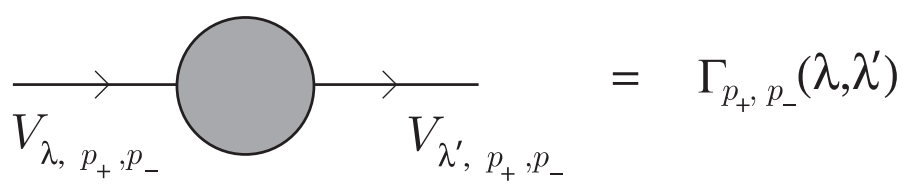

Figure 7: The correction to the free scalar propagator due to interactions. The conserved momenta $p_{ \pm}$flow through the diagram, whereas $\lambda, \lambda^{\prime}$ are the off-shell mass squared of the external legs. The shaded blob is computed using the Feynman rules of section 3 and includes internal propagators winding the compact $y^{-}$ direction.

consider a scalar field with action (17), minimally coupled to gravity, and for simplicity consider the massless case $m=0$. One expects that the existence of CCC's will not allow a consistent definition of single particle states and of an unitary $S$ matrix in the interacting theory. This belief is supported by perturbative computations, since the usual Cutkosky cutting rules are no longer valid, and unitarity fails order by order in the coupling constant [8]. A simple example of this fact will be described in section 4.1 On the other hand, from the duality arguments given in the introduction, one expects, for a given value of the geometric parameter $E$, to be able to define consistently a unitary $S$ matrix for the discrete values of the Planck mass given by (5). For those values of $M$, we expect to be able to define particle states interacting strongly with the gravitational field and scattering with unit probability, as show pictorially in Figure 6

To investigate the possible restoration of unitarity, we shall study the two-point function of the scalar field, which defines single particle states. As shown in Figure 7 this two-point function is determined by the conserved momenta $p_{+}, p_{-}$flowing in the diagram, together with the off-shell mass squared $\lambda, \lambda^{\prime}$ of the external legs. We denote it by $\Gamma_{p_{+}, p_{-}}\left(\lambda, \lambda^{\prime}\right)$, so that the full propagator becomes

$$
16 \pi^{2} E(\lambda+i \epsilon) \delta\left(\lambda-\lambda^{\prime}\right)+\Gamma_{p_{+}, p_{-}}\left(\lambda, \lambda^{\prime}\right) .
$$

It is then possible to define consistently single particle states whenever the above kernel has real eigenfunctions with vanishing eigenvalue. This will certainly be the case if the effective potential $\Gamma_{p_{+}, p_{-}}$determining the propagation of the scalar field satisfies the reality condition

$$
\Gamma_{p_{+}, p_{-}}^{\star}\left(\lambda, \lambda^{\prime}\right)=\Gamma_{p_{+}, p_{-}}\left(\lambda^{\prime}, \lambda\right)
$$

Note that, in quantum field theory, the potential $\Gamma_{p_{+}, p_{-}}$can in principle have an imaginary part coming from on-shell intermediate states. On the other 


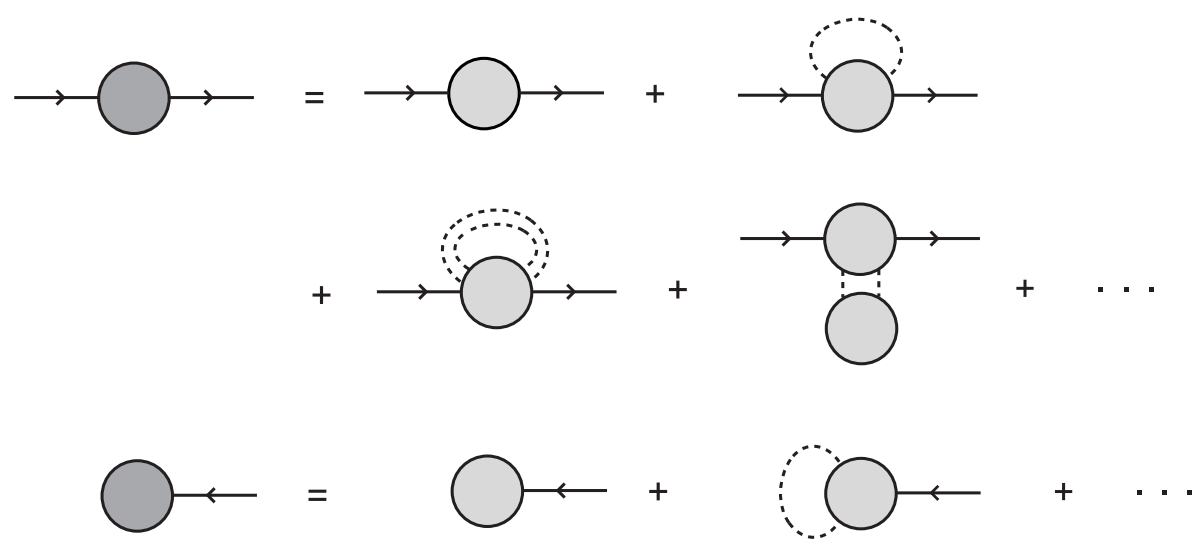

Figure 8: Complete scalar propagator $\Gamma_{p_{+}, p_{-}}\left(\lambda^{\prime}, \lambda\right)$ and tadpole expanded in increasing number of internal propagators with non-vanishing winding number, represented by dashed lines. The remaining effective vertices, shown with light gray blobs, are computed in the parent theory to all orders in the coupling constant, with internal propagators with vanishing winding number.

hand, the contribution to $\Gamma$ considered in this paper has no contributions from intermediate on-shell lines and therefore any deviation from the reality condition (25) would be a sign of inconsistency of the theory. We shall comeback to this point at the end of section 4.4.

We will be able, in particular, to compute the first non-trivial contribution to the full scalar propagator probing the non-causal structure of spacetime. As shown in Figure 8] we consider an expansion of $\Gamma_{p_{+}, p_{-}}\left(\lambda^{\prime}, \lambda\right)$ in increasing number of internal propagators with non-vanishing winding number. We shall focus on the leading non-trivial contribution arising from the graph 9. The loop propagator will have non-vanishing winding number $w$, whereas the bubble in the graph represents the four-point interaction in the parent theory on $\mathbb{M}^{3}$ to all orders in the couplings. In the limit of $p_{+}, \lambda, \lambda^{\prime}$ small, we shall see that, to compute the graph, we will only need control over the parent four-point amplitude in the eikonal kinematical regime, where resummation techniques are known and where general arguments indicate that interactions are dominated by graviton exchange. The form of the eikonal amplitude in three dimensions is such that $\Gamma_{p_{+}, p_{-}}\left(\lambda^{\prime}, \lambda\right)$ satisfies (25) exactly at the values of the gravitational coupling constant $M^{-1}$ given by (5).

As a warm-up exercise, we compute, in the next subsection, the scalar one-loop tadpole graph, so that the reader can get acquainted with loop computations in the orbifold theory. This simple computation already shows 


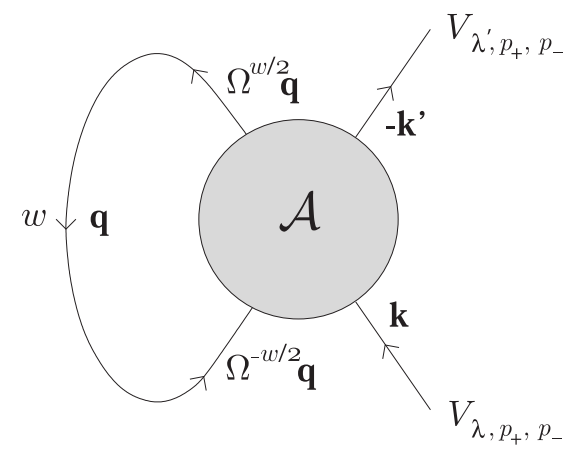

Figure 9: Leading non-trivial contribution to the two-point function $\Gamma_{p_{+}, p_{-}}\left(\lambda, \lambda^{\prime}\right)$, as shown in Figure 8. The loop momentum has non-vanishing winding number $w$, whereas the blob $\mathcal{A}$ represents the four-point amplitude in the parent theory to all orders in the coupling constants.

the breakdown of the cutting rules.

\subsection{One-loop tadpole}

Let us explicitly compute the one-loop tadpole graph of Figure 10, which contributes to the effective action with the term

$$
\int_{\mathcal{F}} d^{3} \mathbf{x} \Phi(\mathbf{x}) \Gamma(\mathbf{x}) .
$$

Conservation of $p_{ \pm}$forces the external leg to have quantum numbers $p_{ \pm}=0$. Thus the one-loop tadpole can be written in momentum space as

$$
\Gamma(p)=\frac{1}{T E^{-1}} \int_{\mathcal{F}} d^{3} \mathbf{x} V_{p, 0}(\mathbf{x}) \Gamma(\mathbf{x}),
$$

where the wave functions $V_{p, 0}$ were introduced in section 2.2. Changing to the $\mathbf{y}$-coordinates, it is easy to see that $\Gamma(\mathbf{y})$ actually depends only on the coordinate $y$, and that

$$
\Gamma(\mathbf{y})=\int \frac{d p}{2 \pi} e^{-i p y} \Gamma(p) \sqrt{\frac{i p}{2 \pi E}} .
$$

To compute the function $\Gamma(p)$, we start with the amplitude $\Gamma(\mathbf{k})$ using Feynman rule $i$ ) of last section

$$
\begin{aligned}
i \Gamma(\mathbf{k})= & \frac{i g}{2} \sum_{w \neq 0} \int \frac{d^{3} q}{(2 \pi)^{3}} \frac{-i}{q^{2}-i \epsilon} e^{\frac{i}{E}\left(w q_{-}+\frac{w^{3}}{24} q_{+}\right)} \times \\
& \times(2 \pi)^{3} \delta\left(k_{+}\right) \delta\left(k_{-}-w q\right) \delta\left(k-w q_{+}\right) \\
= & -2 \pi \delta\left(k_{+}\right) \frac{i g}{2} \sum_{w \neq 0} e^{\frac{i}{E} \frac{w^{2} k}{24}} \int \frac{d q_{-}}{2 \pi i} \frac{e^{\frac{i}{E} w q_{-}}}{2 w q_{-} k-k_{-}^{2}+i \epsilon}
\end{aligned}
$$




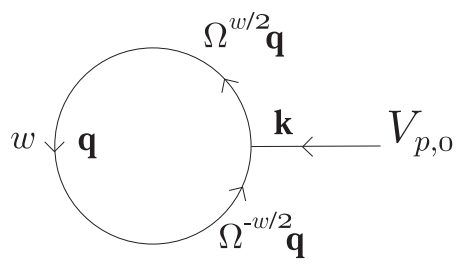

Figure 10: The one-loop scalar tadpole. The on-shell particle winding the compact $y^{-}$direction contributes an imaginary part to the tadpole, which is non-vanishing only for $p<0$. In position space the tadpole diverges at the polarization surfaces.

where the $w=0$ term is eliminated by renormalization of the parent theory. The integral is non-vanishing only for $k<0$. Closing the $q_{-}$counter at infinity, the contribution of the pole, which is associated to the winding particle going on-shell, gives

$$
\Gamma(\mathbf{k})=2 \pi \delta\left(k_{+}\right) \theta(-k) \frac{g}{4|k|} \sum_{w \neq 0} \frac{1}{|w|} e^{\frac{i}{E}\left(\frac{w^{2} k}{24}+\frac{k_{-}^{2}}{2 k}\right)} .
$$

Finally, using (23), we conclude that $T E^{-1} \Gamma(p)=\Gamma(\mathbf{k})$, with $\mathbf{k}=(0,0, p)$, so that the result is

$$
\Gamma(p)=\theta(-p) \frac{g E}{4|p|} \sum_{w \neq 0} \frac{1}{|w|} e^{\frac{i}{E} \frac{w^{2} p}{24}} .
$$

Let us note that, for the simple tadpole graph under consideration, one could compute $\Gamma(\mathbf{x})$ directly in position space

$$
\Gamma(\mathbf{x})=\frac{g}{2} \sum_{w \neq 0} \Delta\left(\Omega^{w} \mathbf{x}, \mathbf{x}\right) .
$$

Denoting with

$$
d_{w}(y)=\sqrt{\frac{2 w^{2}}{E}\left(y-\frac{w^{2}}{24 E}\right)+i \epsilon},
$$

the distance between the point $\mathbf{x}$ and its $w$-th image as a function of the $y$-coordinate $y(\mathbf{x})$, we obtain from the standard expression for the threedimensional scalar propagator

$$
\Gamma(\mathbf{y})=\frac{g}{8 \pi} \sum_{w \neq 0} \frac{1}{d_{w}(y)} .
$$

The reader can check, using (27), that the Fourier transform of the above expression yields (28). 
Clearly, for fixed $w$, the contribution to the one-loop tadpole diverges at the polarization surface $y=w^{2} /(24 E)$ and becomes complex for $y<$ $w^{2} /(24 E)$. Correspondingly, in momentum space, the contribution to $\Gamma(p)$ is non-vanishing only for $p<0$ and comes uniquely from on-shell particles running in the loop, which wind around the compact circle and contribute with an imaginary part to the one-loop tadpole.

In the computation of the graph in Figure 10, the three-vertex is probed at high energies, and therefore interactions will drastically modify its behavior, even in the parent theory. As for the two-point function, we should consider the expansion in Figure 8 and compute graph 10 with the complete parent three-point coupling. This coupling is dominated by gravitational interactions and can be resummed in some cases with eikonal techniques. On the other hand, we shall not pursue this line any further and we shall concentrate mostly on the most relevant computation of the two-point function.

\subsection{Two-point function}

Let us consider now the quadratic term in the effective action, which is composed of the free part in (17), together with the contribution from interactions given by

$$
\frac{1}{2} \int_{\mathcal{F}} d^{3} \mathbf{x} d^{3} \mathbf{x}^{\prime} \Phi(\mathbf{x}) \Phi\left(\mathbf{x}^{\prime}\right) \Gamma\left(\mathbf{x}, \mathbf{x}^{\prime}\right)
$$

In momentum space the two-point function $\Gamma\left(\mathbf{x}, \mathbf{x}^{\prime}\right)$ becomes

$$
\begin{aligned}
\int_{\mathcal{F}} d^{3} \mathbf{x} d^{3} \mathbf{x}^{\prime} V_{\lambda, p_{+}, p_{-}}(\mathbf{x}) V_{\lambda^{\prime}, p_{+}^{\prime}, p_{-}^{\prime}}\left(\mathbf{x}^{\prime}\right) \Gamma\left(\mathbf{x}, \mathbf{x}^{\prime}\right)= \\
=E^{-1} \delta_{p_{-}+p_{-}^{\prime}} 2 \pi \delta\left(p_{+}+p_{+}^{\prime}\right) \Gamma_{p_{+}, p_{-}}\left(\lambda, \lambda^{\prime}\right)
\end{aligned}
$$

where $\Gamma_{p_{+}, p_{-}}\left(\lambda, \lambda^{\prime}\right)=\Gamma_{-p_{+},-p_{-}}\left(\lambda^{\prime}, \lambda\right)$. As for the one-loop tadpole, the last equation can be explicitly computed using the Feynman rules of section 3. in particular using equation (21). It gives the two-point function written in the basis $V_{\lambda, p_{+}, p_{-}}$, with the conservation of $p_{ \pm}$momenta explicitly stated. Reality of the interaction $\Gamma\left(\mathbf{x}, \mathbf{x}^{\prime}\right)$ then reads

$$
\Gamma_{p_{+}, p_{-}}^{\star}\left(\lambda, \lambda^{\prime}\right)=\Gamma_{p_{+}, p_{-}}\left(\lambda^{\prime}, \lambda\right)=\Gamma_{-p_{+},-p_{-}}\left(\lambda, \lambda^{\prime}\right)
$$

Using (21), we may write $\Gamma_{p_{+}, p_{-}}\left(\lambda, \lambda^{\prime}\right)$ as follows

$$
\Gamma_{p_{+}, p_{-}}\left(\lambda, \lambda^{\prime}\right)=\frac{2 E}{T\left|p_{+}\right|} \int d k e^{i \chi(k)} \Gamma\left(\mathbf{k}, \mathbf{k}^{\prime}\right),
$$


where the phase $\chi(k)$ is explicitly given by

$$
\chi(k)=\frac{1}{E p_{+}^{2}}\left[\left(2 p_{+} p_{-}-\frac{\lambda+\lambda^{\prime}}{2}\right) k-\frac{k^{3}}{3}\right] .
$$

The amplitude $\Gamma\left(\mathbf{k}, \mathbf{k}^{\prime}\right)$ is computed using Feynman rule $\left.i i\right)$ of section 3, with external momenta given by

$$
\mathbf{k}=\left(p_{+}, \frac{\lambda+k^{2}}{2 p_{+}}, k\right), \quad \mathbf{k}^{\prime}=\left(-p_{+},-\frac{\lambda^{\prime}+k^{2}}{2 p_{+}}, k\right) .
$$

We now focus our attention on external states in the two-point function such that the integral (31) can be computed using the saddle point approximation. This requires that $4 p_{+} p_{-}>\lambda+\lambda^{\prime}$, in order to have saddle points on the real $k$ axis at $k= \pm p$, with

$$
p=\sqrt{2 p_{+} p_{-}-\frac{\lambda+\lambda^{\prime}}{2}} .
$$

Then, the gaussian approximation to (31) is valid provided that

$$
E p_{+}^{2} \ll p^{3}
$$

In this situation, the two-point function becomes

$$
\Gamma_{p_{+}, p_{-}}\left(\lambda, \lambda^{\prime}\right) \simeq \frac{E}{T} \sqrt{\frac{4 \pi E}{i p}} e^{\frac{2 i}{3} \frac{p^{3}}{E p_{+}^{2}}} \Gamma\left(\mathbf{k}, \mathbf{k}^{\prime}\right)+(p \leftrightarrow-p),
$$

where the external momenta $\mathbf{k}, \mathbf{k}^{\prime}$ are fixed, at the saddle point $k=p$, to be

$$
\mathbf{k}=\left(p_{+}, p_{-}+\frac{\lambda-\lambda^{\prime}}{4 p_{+}}, p\right), \quad \quad \mathbf{k}^{\prime}=\left(-p_{+},-p_{-}+\frac{\lambda-\lambda^{\prime}}{4 p_{+}}, p\right) .
$$

We consider in what follows the first non-trivial two-point graph probing the non-causal structure of space-time, which has a single scalar propagator with non-vanishing winding number $w$. This graph is shown in Figure 9 where the bubble represents the four-point amplitude in the parent theory which we denote by

$$
(2 \pi)^{3} \delta^{3}\left(\Omega^{-w / 2} \mathbf{q}+\mathbf{k}+\mathbf{k}^{\prime}-\Omega^{w / 2} \mathbf{q}\right) \mathcal{A}\left(\Omega^{-w / 2} \mathbf{q}, \mathbf{k}, \mathbf{k}^{\prime},-\Omega^{w / 2} \mathbf{q}\right) .
$$

Since $\left(\Omega^{-w / 2} \mathbf{q}\right)^{2}=\left(\Omega^{w / 2} \mathbf{q}\right)^{2}$, the amplitude $\mathcal{A}$ depends only on five kinematical invariants. Moreover, since $\lambda=-\mathbf{k}^{2}$ and $\lambda^{\prime}=-\mathbf{k}^{\prime 2}$ are kept fixed, 
$\mathcal{A}$ depends only on the Mandelstam invariants

$$
\begin{aligned}
s & =-\left(\mathbf{k}+\Omega^{-w / 2} \mathbf{q}\right)^{2} \\
t & =-\left(\mathbf{k}+\mathbf{k}^{\prime}\right)^{2} \\
u & =-\left(\mathbf{k}-\Omega^{w / 2} \mathbf{q}\right)^{2}
\end{aligned}
$$

Finally, again because $\left(\Omega^{-w / 2} \mathbf{q}\right)^{2}=\left(\Omega^{w / 2} \mathbf{q}\right)^{2}$, the amplitude $\mathcal{A}(s, t, u)$ is symmetric under interchange of $s \leftrightarrow u$ and of $\lambda \leftrightarrow \lambda^{\prime}$.

For simplicity, we consider first the case of on-shell external states with $\lambda=\lambda^{\prime}=0$. In this case, in order to satisfy (32), we will take the limit of small $p_{+}$, since $p_{-}$and $E$ are fixed. Then, the contribution to $\Gamma\left(\mathbf{k}, \mathbf{k}^{\prime}\right)$ of the graph with winding number $w$ is

$$
\begin{aligned}
\Gamma_{w} & \left(\mathbf{k}, \mathbf{k}^{\prime}\right)=\frac{1}{2} \int \frac{d^{3} \mathbf{q}}{(2 \pi)^{3}} \frac{i}{-\mathbf{q}^{2}+i \epsilon} e^{\frac{i}{E}\left(w q_{-}+\frac{w^{3}}{24} q_{+}\right)} \times \\
& \times(2 \pi)^{3} \delta^{3}\left(\Omega^{-w / 2} \mathbf{q}+\mathbf{k}+\mathbf{k}^{\prime}-\Omega^{w / 2} \mathbf{q}\right) \mathcal{A}\left(\Omega^{-w / 2} \mathbf{q}, \mathbf{k}, \mathbf{k}^{\prime},-\Omega^{w / 2} \mathbf{q}\right) \\
= & -\frac{T}{8} e^{\frac{i}{E} \frac{w^{2} p}{12}} \int \frac{d q_{-}}{2 \pi i} \frac{1}{p w q_{-}+i \epsilon} e^{\frac{i}{E} w q_{-}} \mathcal{A}\left(\Omega^{-w / 2} \mathbf{q}, \mathbf{k}, \mathbf{k}^{\prime},-\Omega^{w / 2} \mathbf{q}\right) .
\end{aligned}
$$

Two of the $\delta$-functions fix $q_{+}=2 p / w$ and $q=0$, while the remaining $\delta$-function gives the overall factor $T$. In the last line, we therefore have that

$$
\begin{aligned}
\Omega^{-w / 2} \mathbf{q} & =\left(\frac{2 p}{w}, q_{-}+\frac{w p}{4},-p\right), \\
-\Omega^{w / 2} \mathbf{q} & =-\left(\frac{2 p}{w}, q_{-}+\frac{w p}{4}, p\right) .
\end{aligned}
$$

The corresponding Mandelstam invariants can be readily computed

$$
\begin{aligned}
s & =2\left(\frac{2 p}{w}+p_{+}\right)\left(q_{-}+\frac{w p}{4}+p_{-}\right) \simeq \frac{4 p}{w}\left(q_{-}+p_{-}\right) \\
t & =-4 p^{2} \\
u & =2\left(\frac{2 p}{w}-p_{+}\right)\left(q_{-}+\frac{w p}{4}-p_{-}\right) \simeq \frac{4 p}{w}\left(q_{-}-p_{-}\right)
\end{aligned}
$$

where we have expanded $s$ and $u$ to leading order in $p \sim \sqrt{\left|p_{+}\right|}$, since we are working in the limit $p_{+} \rightarrow 0$. More precisely, we will be working under the assumption that

$$
p \ll \frac{E}{|w|},
$$

which implies (32) but is more stringent for $|w| \gg 1$. 
The integral (33) reads

$$
\Gamma_{w}\left(\mathbf{k}, \mathbf{k}^{\prime}\right) \simeq-\frac{T}{8} e^{\frac{i}{E} \frac{w^{2} p}{12}} \int \frac{d q_{-}}{2 \pi i} \frac{1}{p w q_{-}+i \epsilon} e^{\frac{i}{E} w q_{-}} \mathcal{A}(s, t, u)
$$

where the Mandelstam invariants are given above. Notice that this integral is invariant under $w \rightarrow-w$. This can be seen by changing integration variable to $-q_{-}$and by using $s \leftrightarrow u$ invariance of $\mathcal{A}$. For the same reasons, changing $p \rightarrow-p$ has the unique effect of changing the phases to their complex conjugates $e^{-\frac{i}{E} w q_{-}}$and $e^{-\frac{i}{E} \frac{w^{2} p}{12}}$. Using (31), we conclude that the two-point amplitude can be written as

$$
\Gamma_{p_{+}, p_{-}}(0,0) \simeq \sum_{w \neq 0}\left(c_{w} \Gamma_{w}^{+}+c_{w}^{\star} \Gamma_{w}^{-}\right)
$$

where

$$
\Gamma_{w}^{ \pm}=\int \frac{d s}{2 \pi i} \frac{e^{ \pm \frac{i}{4} \frac{w^{2}}{p E} s}}{|w| s-4 p p_{-}+i \epsilon} \mathcal{A}\left(s, t=-4 p^{2}, u=s-\frac{8 p p_{-}}{|w|}\right)
$$

and the constant $c_{w}$ is given by

$$
c_{w}=-\frac{E}{8 p} \sqrt{\frac{4 \pi E}{i p}} e^{\frac{i}{E}\left(\frac{2 p^{3}}{3 p_{+}^{2}}+\frac{w^{2} p}{12}\right)} .
$$

The reality condition (30) implies that $\Gamma_{p_{+}, p_{-}}(0,0)$ is real, which in turn implies that $\left(\sum_{w} c_{w} \Gamma_{w}^{+}\right)^{\star}=\sum_{w} c_{w}^{\star} \Gamma_{w}^{-}$. On physical grounds, we expect the stronger condition

$$
\left(\Gamma_{w}^{+}\right)^{\star}=\Gamma_{w}^{-}
$$

to hold for each value of $w$. This can be understood, for example, from the behavior of the one-loop tadpole in position space. For a given value of $w$, the contribution to the tadpole diverges and acquires an imaginary part at the $w$-th polarization surface. Therefore, in position space, this singular behavior occurs at widely separated positions for different values of the winding number $w$ and we expect these pathologies to be cured for each $w$.

We close this section with an important kinematic consideration. When the winding particle is on-shell, then $s=-u=4 p p_{-} /|w|$. Since $|t| \sim p^{2}$, the parent amplitude is evaluated in the eikonal regime

$$
|s|,|u| \gg|t| .
$$


Moreover, this regime continues to be valid throughout the whole integration region in $s$. In fact, for the non-generic case where $|s| \lesssim|t|$, the other invariant $u$ is of order $p p_{-} /|w|$ and still satisfies $u \gg t$. A similar remark applies when $|u| \lesssim|t|$. In the following section we do a detour on the eikonal approximation to the scattering of a scalar field in three dimensions. The results will be used in the computation of the orbifold two-point amplitude.

\subsection{Eikonal approximation}

Let us recall first the $s$-channel partial wave decomposition for the on-shell scattering amplitude of massless scalars in three dimensions

$$
1+i \mathcal{A}=4 \sqrt{s} \sum_{n} e^{i n \theta} e^{2 i \delta_{n}(s)} .
$$

The scattering angle $\theta$ is given by $\sin ^{2}(\theta / 2)=-t / s$ and the phase shifts satisfy $\delta_{n}=\delta_{-n}$. Unitarity requires that $\operatorname{Im} \delta_{n} \geq 0$. In the eikonal limit $s \simeq-u \gg-t$, the scattering angle is given by $\theta \simeq 2 \sqrt{-t / s}$ and one may replace the sum over partial waves with an integral over the impact parameter $x=2 n / \sqrt{s}$. One then obtains the eikonal expression

$$
1+i \mathcal{A} \simeq 2 s \int d x e^{i x \sqrt{-t}} e^{2 i \delta(s, x)}
$$

This representation can be derived by studying the $s \gg|t|$ limit of the generalized ladder graphs shown in Figure [1]. It turns out that the loop expansion matches the expansion in powers of $\delta$ [7, 33, 34, 35], so that the phase shift is given, in terms of the leading tree-level interaction $\mathcal{A}_{\text {tree }}(s, t)$, by the simple Fourier transform

$$
\mathcal{A}_{\text {tree }} \simeq 4 s \int d x e^{i x \sqrt{-t}} \delta(s, x) .
$$

Consider now scattering due to the exchange of a spin $j$ massless particle. Then $\mathcal{A}_{\text {tree }} \simeq-4 M^{3-2 j} s^{j} / t$, where we are implicitly assuming that the coupling constants are of order one in Planck units ${ }^{3}$. The phase shift is then given by

$$
2 \delta(s, x) \simeq-\left(s / M^{2}\right)^{j-1} M|x| .
$$

\footnotetext{
${ }^{3}$ We assume that all couplings are of the same order in Planck units mostly for notational simplicity. In case of a large ratios between the couplings, the gravitational interaction will still dominate in the regimes of interest, as explained in the rest of this section, but the specific bounds will have to be modified accordingly. Moreover, the most relevant examples coming from compactifications of supergravity theories do have a single gravitational coupling.
} 


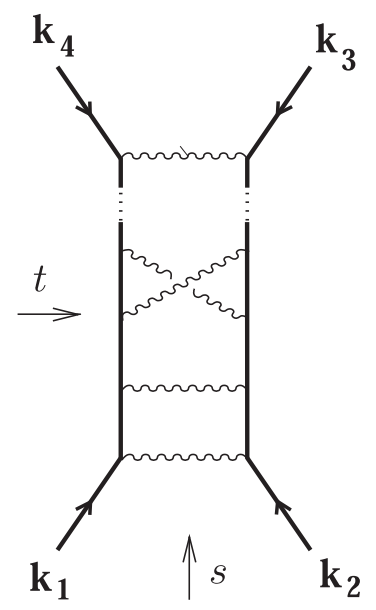

Figure 11: Generalized ladder diagram contributing to the scattering amplitude $\mathcal{A}$ of scalar particles in the eikonal regime $s \gg|t|$. The exchanged particle can have in general spin $j$ but, in the kinematical regime of interest, the gravitational interaction $j=2$ will dominate.

It is linear in the impact parameter $|x|$ and negative. These facts are easily understood in the $j=2$ case of graviton exchange. In this case, following the work of 't Hooft [7], we can think of the scattered particle as moving in the conical geometry created by the target. The qualitative features of (40) can then be immediately understood from Figure 12. The full eikonal amplitude for spin $-j$ exchange finally reads 36, 37, 38.

$$
1+i \mathcal{A} \simeq-4 i M \frac{\left(s / M^{2}\right)^{j}}{\left(t / M^{2}\right)+\left(s / M^{2}\right)^{2 j-2}-i \epsilon} .
$$

This result has been quoted in the literature only for the case $j=2$, although its derivation easily extends to the case of general $j$, as shown in the above derivation.

The eikonal amplitude has a pole at real values of the kinematical invariants given by $-t=M^{2}\left(s / M^{2}\right)^{2 j-2}$. We shall call this pole the 't Hooft pole, in analogy with those discussed in [7] for graviton exchange in four dimensions. At the pole, the $i \epsilon$ prescription is obtained by requiring convergence of the integral (39) at large values of $|x|$. One can physically understand this prescription by first noting that, at vanishing coupling and phase-shift, (39) gives the $S$-matrix element

$$
4 \pi s \delta(\sqrt{-t}) \text {. }
$$

This amplitude corresponds to free propagation of particles, with no inter- 


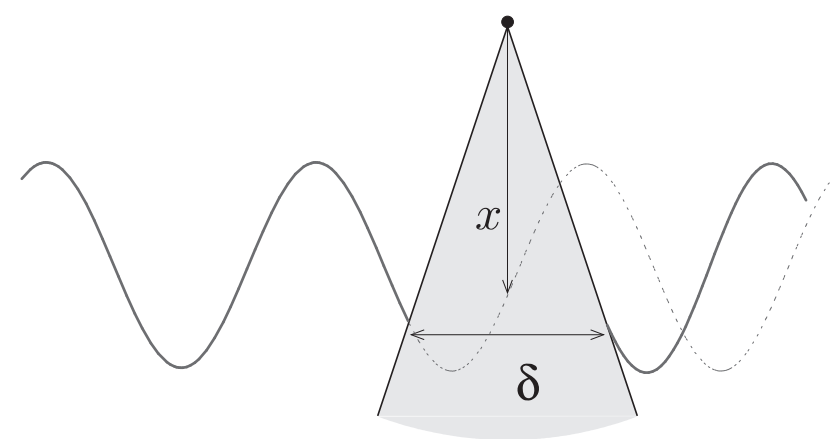

Figure 12: Phase shift for a scattering process in the gravitational background created by the target. In three dimensions, the background geometry is a conical space with deficit angle proportional to $\sqrt{s} / M$. The phase shift $\delta$ is proportional to the impact parameter $x$ and is negative.

action. Using the fact that $\operatorname{Im}(x-i \epsilon)^{-1}=\pi \delta(x)$ in the amplitude (41), we then see that the $\delta$-function contribution in the free theory is replaced, in the interacting theory, by

$$
2 \pi s \delta\left(\sqrt{-t}-M\left(s / M^{2}\right)^{j-1}\right)+2 \pi s \delta\left(\sqrt{-t}+M\left(s / M^{2}\right)^{j-1}\right) .
$$

We conclude that a specific scattering angle, dependent on the energy of the process, is singled out by the eikonal amplitude. Notice that the pole is in the physical eikonal region $s \gg-t$ whenever $\left(s / M^{2}\right)^{2 j-3} \ll 1$. This shows that gravitons behave quite differently from lower spin particles. In fact, for $j=2$, the eikonal approximation is reliable around the 't Hooft pole for center of mass energies well below the Planck mass.

We may now discuss which interaction dominates in the kinematical regime of interest in (36). More specifically, we will concentrate on the cases $j=0,1,2$, which arise in standard compactifications of supergravity theories. We will compare the relative importance of the various contributions when the propagator in (36) is on-shell, that is at $s=4 p p_{-} /|w|$ and $t=-4 p^{2}$. We are only interested in an order of magnitude estimate, dropping factors of order unity. Assume that the Kałuża-Klein charge $n=p_{-} /(2 \pi E)$ is of order unity and that

$$
E \sim \frac{M}{J}
$$

with $J \gtrsim 1$, which is consistent with the quantization condition (15). The kinematical invariants are then given by

$$
s \sim \frac{p E}{w} \sim \frac{p M}{w J}, \quad|t| \sim p^{2},
$$


and the basic requirement $s \gg|t|$ for the eikonal approximation to be valid is

$$
p \ll \frac{M}{w J} .
$$

When this condition is satisfied, the saddle point approximation (32) in the previous subsection is also justified.

Next we analyze the behavior of the eikonal amplitude (41) for different values of $j$. For $j=0,1$, the denominator in (41) is dominated by the second term, whereas for $j=2$ the first term dominates. We therefore arrive at the following estimates for the amplitudes

$$
\begin{array}{rlrl}
M\left(s / M^{2}\right)^{2-j} & \sim M\left(\frac{p}{M w J}\right)^{2-j} \ll \frac{M}{(w J)^{4-2 j}}, & (j=0,1) \\
\frac{1}{M} \frac{s^{2}}{t} & \sim \frac{M}{(w J)^{2}} . & & (j=2)
\end{array}
$$

Thus, the graviton interaction dominates in the kinematical region of interest. Therefore, from now on, we will only consider the case $j=2$.

Up to this point, we have discussed the amplitude $\mathcal{A}$ on-shell, with $s \simeq-u$. On the other hand, as it is clear from (36), one needs to understand the off-shell extension of the amplitude (41) for generic values of $s, u$. We also allow in principle for non-vanishing $\lambda, \lambda^{\prime}$, small compared to $s, u$. In what follows, we shall indicate explicitly only the dependence on the "large" variables $s, u$, leaving implicit the dependence on the "small" ones $t, \lambda, \lambda^{\prime}$. The tree level result is simple to compute and gives

$$
\mathcal{A}_{\text {tree }} \simeq-\frac{(s-u)^{2}}{M t} .
$$

To understand the off-shell amplitude to all orders, we first rewrite the on-shell result (41) as follows

$$
\mathcal{A}_{+} \simeq-\frac{(s-u)^{2}}{2 \sqrt{-t}}\left(\frac{1}{s-M \sqrt{-t}-i \epsilon}+\frac{1}{u-M \sqrt{-t}-i \epsilon}\right) .
$$

We neglect from now on the free propagation term $4 \pi s \delta(\sqrt{-t})$ in the $S$ matrix element (41) since it does not contribute to the orbifold amplitude (36), which is computed at fixed non-vanishing momentum transfer. One may interpret (43) as the exchange, in the $s$ and $u$ channels, of an effective particle of mass squared $M \sqrt{-t}$. Note, however, that the $i \epsilon$ prescription, which arises from the eikonal result, is opposite to the usual one. Alternatively, recalling that $s \simeq-u$ on-shell, one rewrites (43) as

$$
\mathcal{A}_{-} \simeq \frac{(s-u)^{2}}{2 \sqrt{-t}}\left(\frac{1}{s+M \sqrt{-t}+i \epsilon}+\frac{1}{u+M \sqrt{-t}+i \epsilon}\right)
$$


which can be viewed as resulting from the exchange of a tachyonic effective particle of mass squared $-M \sqrt{-t}$, with the usual $i \epsilon$ prescription.

Given the above observations, we shall assume that the off-shell extension of the eikonal amplitude has poles determined by effective particles with mass squared $\pm M \sqrt{-t}$. The amplitude $\mathcal{A}(s, u)$ therefore has poles placed at

$$
s=M \sqrt{-t}+i \epsilon, \quad u=M \sqrt{-t}+i \epsilon,
$$

and at

$$
s=-M \sqrt{-t}-i \epsilon, \quad u=-M \sqrt{-t}-i \epsilon .
$$

We denote the residues of the amplitude $\mathcal{A}(s, u)$ by

$$
\begin{aligned}
& \operatorname{Res}_{s} \mathcal{A}(s= \pm M \sqrt{-t}, u=s-\sigma)=f_{ \pm}(\sigma), \\
& \operatorname{Res}_{u} \mathcal{A}(s=u+\sigma, u= \pm M \sqrt{-t})=f_{ \pm}(-\sigma),
\end{aligned}
$$

where the real functions $f_{ \pm}$are the same at the $s$ and $u$ poles because of $s, u$ symmetry of the amplitude. For later convenience, we expressed the above functions $f_{ \pm}$in terms of $\sigma=s-u$.

It is important to stress that, in order to compute the orbifold two-point function and to check the quantization condition (15), we shall only assume that the poles of the eikonal amplitude are placed at $s, u= \pm M \sqrt{-t}$, as for the on-shell case. The $i \epsilon$ prescription, on the other hand, is fixed by the on-shell computation. Finally, the reality of the functions $f_{ \pm}$follows from the fact that, in the absence of discontinuities, field theoretic amplitudes are real.

A simple example of an off-shell extension of the eikonal amplitude (41), satisfying the above requirements, is

$$
a_{+} \mathcal{A}_{+}+a_{-} \mathcal{A}_{-}
$$

with $a_{ \pm}$constant and $\mathcal{A}_{ \pm}$as defined above. To match the off-shell tree-level result (42) and the on-shell eikonal amplitude we must have that

$$
a_{+}+a_{-}=1
$$

\subsection{Quantization condition from unitarity}

We are now in position to finish the computation of the two-point function for a massless scalar field in the orbifold geometry. As shown in sections 4.2 


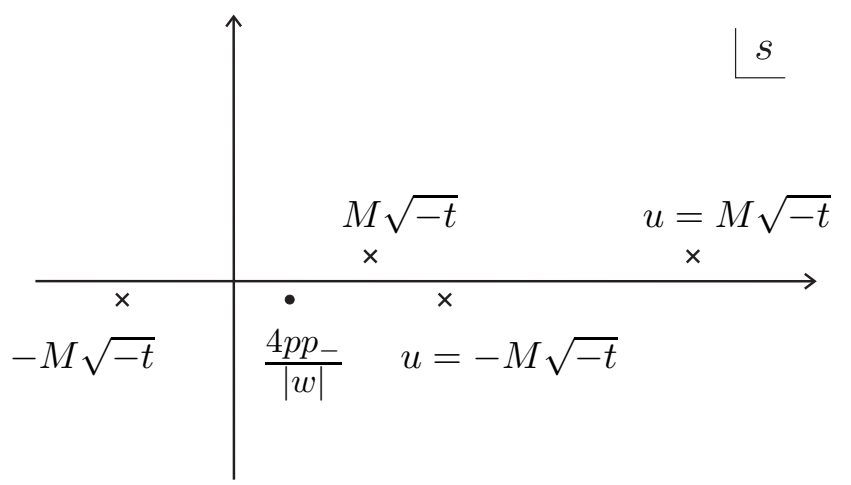

Figure 13: Poles of the integrand in equation (46) in the $s$-plane. The pole denoted with a dot comes from the winding propagator, whereas the poles marked with a cross come from the eikonal amplitude $\mathcal{A}$. Recall that $s=u+\sigma$, with $\sigma=8 p p_{-} /|w|$.

and 4.3, this amounts to evaluating the integral

$$
\Gamma_{w}^{ \pm}=\int \frac{d s}{2 \pi i} \frac{e^{ \pm \frac{i}{4} \frac{w^{2}}{p E} s}}{|w| s-4 p p_{-}+i \epsilon} \mathcal{A}\left(s, t=-4 p^{2}, u=s-\frac{8 p p_{-}}{|w|}\right)
$$

where the amplitude $\mathcal{A}$ is dominated by graviton exchange in the eikonal regime. The integrand has a pole at $s=4 p p_{-} / w-i \epsilon$, coming from the winding propagator, and poles at $s= \pm M \sqrt{-t} \pm i \epsilon$ and at $u= \pm M \sqrt{-t} \pm i \epsilon$, from the eikonal amplitude $\mathcal{A}$. The pole structure of the integrand is shown in Figure 13

To compute $\Gamma_{w}^{+}$one closes the $s$-contour in the upper half plane, so that the integral is determined by the poles of $\mathcal{A}$ with positive imaginary part at $s, u=M \sqrt{-t}+i \epsilon=2 p M+i \epsilon$. The phase in (46) is given at both poles by $e^{i \frac{w^{2} M}{2 E}}$, since $p_{-}$is quantized in units of $2 \pi E$. One then obtains

$$
\Gamma_{w}^{+}=\frac{1}{|w|} e^{i \frac{w^{2} M}{2 E}} F_{+}
$$

where $F_{+}$is given by the real constant

$$
F_{+}=\frac{2 f_{+}(\sigma)}{4 p M-\sigma}+\frac{2 f_{+}(-\sigma)}{4 p M+\sigma}
$$

and where $\sigma=s-u=8 p p_{-} /|w|$. Similarly, $\Gamma_{w}^{-}$is determined by the poles in the lower half $s$-plane, with the result

$$
\Gamma_{w}^{-}=\frac{1}{|w|}\left(e^{i \frac{w^{2} M}{2 E}} F_{-}-\mathcal{A}_{\text {on-shell }}\right)
$$


where now

$$
F_{-}=\frac{2 f_{-}(\sigma)}{4 p M+\sigma}+\frac{2 f_{-}(-\sigma)}{4 p M-\sigma} .
$$

The on-shell amplitude

$$
\mathcal{A}_{\mathrm{on}-\mathrm{shell}}=-4 M \frac{4 p_{-}^{2}}{4 p_{-}^{2}-w^{2} M^{2}}=\frac{4 M \sigma^{2}}{16 M^{2} p^{2}-\sigma^{2}}
$$

is computed at the pole of the winding propagator.

Finally, we can investigate the implication of the reality condition (38), which now reads

$$
-e^{i \frac{w^{2} M}{2 E}} F_{+}+e^{-i \frac{w^{2} M}{2 E}} F_{-}=\mathcal{A}_{\text {on-shell }} .
$$

We consider the equation above for different values of $w$. In order for the inequality (35) to be valid for all $w$, we keep $p_{-}$fixed and tune $p_{+}$so that $p=\Lambda /|w|$, for a fixed energy scale $\Lambda \ll E$. As $w \rightarrow \infty$, we then have that $\sigma=8 \Lambda p_{-} / w^{2} \rightarrow 0$. Note that $F_{+}, F_{-}$and $\mathcal{A}_{\text {on-shell }}$ are all analytic functions of $\sigma$, with at most poles and branch cuts. This is clear for (47), and it follows from general properties of analyticity of Feynman amplitudes for the functions $f_{ \pm}$, and therefore for $F_{ \pm}$. As $w \rightarrow \infty$, the phase $e^{i \frac{w^{2} M}{2 E}}$ oscillates unless $e^{i \frac{M}{2 E}}=1$, whereas the functions $F_{+}, F_{-}$and $\mathcal{A}_{\text {on-shell }}$ have a regular behavior for $\sigma \rightarrow 0$. Under mild regularity assumptions, we show ${ }^{4}$ in appendix $\mathrm{B}$ that, in order for (48) to be satisfied for all values of $w$, we must have that $e^{i \frac{M}{2 E}}=1$ and therefore that

$$
\frac{M}{2 E} \in 2 \pi \mathbb{Z},
$$

which is the quantization condition (5). In this case, we have the additional requirement on the residues

$$
-F_{+}+F_{-}=\mathcal{A}_{\text {on-shell }}
$$

Consider this last constraint in the case of the off-shell eikonal amplitude given in example (44). The residue functions $f_{ \pm}$are then explicitly given by

$$
f_{ \pm}(\sigma)=\mp a_{ \pm} \frac{\sigma^{2}}{4 p}
$$

and we conclude that $F_{ \pm}=\mp a_{ \pm} \mathcal{A}_{\text {on-shell }}$. Therefore, condition (49) implies that $a_{+}+a_{-}=1$, exactly as in (45).

\footnotetext{
${ }^{4}$ We thank M. Cornalba for suggesting this argument.
} 
A few comments are in order. Firstly note that, in the regime of interest, all kinematical invariants are much smaller than $M / J$. Assuming that the three dimensional geometry comes from a ten dimensional string compactification with Planck and string masses, respectively given by $M_{10}$ and $M_{s}$, we see that string and Kałuża-Klein effects are irrelevant when $J M_{s} \gg M$ and when $J^{7 / 8} M_{10} \gg M$, which is always true for large charge $J$.

Secondly, suppose we use, instead of the full eikonal amplitude $\mathcal{A}$, only the tree level result $-4 s^{2} / t M$ for gravitational scattering. Then, the single contribution to the two-point function comes from the pole of the winding propagator, violating the reality condition (38). This one-loop violation of unitarity is analogous to the one found in the computation of the one-point function in section 4.1, where the tadpole $\Gamma(p)$ in (28) has support only for $p<0$.

Thirdly, within the eikonal approximation we cannot have a violation of the reality of $\Gamma_{p_{+}, p_{-}}$due to intermediate on-shell lines. In fact, the eikonal interaction is essentially transverse 33, with no $k_{+}$exchange. Therefore, the scalar lines in Figure 1 have a fixed value of the $K_{+}$momentum. It is then impossible to cut the graph so that all the cut propagators have positive $K_{+}$momentum flowing from one part of the graph to the other.

Finally, note that, although the amplitude (44) is the simplest off-shell generalization of the on-shell eikonal result, it is quite remarkable that we obtain the same constraint on the coefficients $a_{ \pm}$by imposing the condition (49) on the residues. This fact clearly deserves a more thorough investigation, which we leave for future work.

\section{Extending the results to off-shell external states}

We now generalize the results of the last section to the case of non-vanishing $\lambda, \lambda^{\prime}$. More precisely, we shall work in the limit when $E p_{+} \gtrsim \lambda, \lambda^{\prime}$ satisfying

$$
E p_{+}, \lambda, \lambda^{\prime} \ll(E / w)^{2},
$$

where $w$ is the winding number of the loop propagator in Figure 9 The first expression in equation (33) is still correct, but now the $\delta$-functions fix $q_{+}=2 p / w$ and $q=\left(\lambda-\lambda^{\prime}\right) /\left(2 w p_{+}\right)$. Therefore we have that

$$
\begin{aligned}
\Gamma_{w}\left(\mathbf{k}, \mathbf{k}^{\prime}\right)=-\frac{T}{8} e^{\frac{i}{E} \frac{w^{2} p}{12}} \int \frac{d q_{-}}{2 \pi i} \frac{1}{p w q_{-}-\frac{\left(\lambda-\lambda^{\prime}\right)^{2}}{16 p_{+}^{2}}+i \epsilon} \times \\
\times e^{\frac{i}{E} w q_{-}} \mathcal{A}\left(\Omega^{-w / 2} \mathbf{q}, \mathbf{k}, \mathbf{k}^{\prime},-\Omega^{w / 2} \mathbf{q}\right),
\end{aligned}
$$


with

$$
\begin{aligned}
\Omega^{-w / 2} \mathbf{q} & =\left(\frac{2 p}{w}, q_{-}-\frac{\lambda-\lambda^{\prime}}{4 p_{+}}+\frac{w p}{4}, \frac{\lambda-\lambda^{\prime}}{2 w p_{+}}-p\right) \\
-\Omega^{w / 2} \mathbf{q} & =-\left(\frac{2 p}{w}, q_{-}+\frac{\lambda-\lambda^{\prime}}{4 p_{+}}+\frac{w p}{4}, \frac{\lambda-\lambda^{\prime}}{2 w p_{+}}+p\right) .
\end{aligned}
$$

An approximation similar to the one used in equation (34) gives the Mandelstam invariants

$$
\begin{aligned}
s & \simeq \frac{4 p}{w}\left(q_{-}+p_{-}\right)-\frac{\left(\lambda-\lambda^{\prime}\right)^{2}}{4 w^{2} p_{+}^{2}} \\
t & =-4 p^{2} \\
u & \simeq \frac{4 p}{w}\left(q_{-}-p_{-}\right)-\frac{\left(\lambda-\lambda^{\prime}\right)^{2}}{4 w^{2} p_{+}^{2}} .
\end{aligned}
$$

Just as for the on-shell case, the expression for $\Gamma_{w}\left(\mathbf{k}, \mathbf{k}^{\prime}\right)$ is invariant under $p \leftrightarrow-p$, aside from conjugation of the phase. Moreover, $\Gamma_{w}\left(\mathbf{k}, \mathbf{k}^{\prime}\right)$ is invariant under $w \leftrightarrow-w$ and $\lambda \leftrightarrow \lambda^{\prime}$. Thus we arrive at the final expression

$$
\Gamma_{p_{+}, p_{-}}\left(\lambda, \lambda^{\prime}\right) \simeq e^{\frac{i}{E} \frac{\left(\lambda-\lambda^{\prime}\right)^{2}}{16 p_{+}^{2} p}} \sum_{w \neq 0} c_{w} \Gamma_{w}^{+}+e^{-\frac{i}{E} \frac{\left(\lambda-\lambda^{\prime}\right)^{2}}{16 p_{+}^{2} p}} \sum_{w \neq 0} c_{w}^{\star} \Gamma_{w}^{-},
$$

where the constant $c_{w}$, given by (37), is unchanged from the on-shell case and where

$$
\Gamma_{w}^{ \pm}=\int \frac{d s}{2 \pi i} \frac{e^{ \pm \frac{i}{4} \frac{w^{2}}{p E} s}}{|w| s-4 p p_{-}+i \epsilon} \mathcal{A}\left(s, t=-4 p^{2}, u=s-\frac{8 p p_{-}}{|w|}\right) .
$$

The expression for $\Gamma_{w}^{ \pm}$is formally identical to the on-shell expression (36), but yields a result depending on the external off-shell masses, due to the implicit dependence of the momentum $p$ and of the amplitude $\mathcal{A}$ on $\lambda, \lambda^{\prime}$. As for the on-shell case, the reality condition (30), together with the fact that $\Gamma_{p_{+}, p_{-}}\left(\lambda, \lambda^{\prime}\right)$ is symmetric in $\lambda, \lambda^{\prime}$, implies that $\left(\sum c_{w} \Gamma_{w}^{+}\right)^{\star}=\sum c_{w}^{\star} \Gamma_{w}^{-}$. Under the usual assumption regarding the separation of different winding modes, we have that $\left(\Gamma_{w}^{+}\right)^{\star}=\Gamma_{w}^{-}$.

\section{$5.1 \quad$ Particle states}

To conclude the discussion of the two-point function, we wish to analyze the single particle wave function $\Psi(\lambda)$, which solves the linearized quantum 
equation of motion

$$
\int d \lambda^{\prime} K\left(\lambda, \lambda^{\prime}\right) \Psi\left(\lambda^{\prime}\right)=0
$$

The kernel $K$ is given by the full propagator (24)

$$
K\left(\lambda, \lambda^{\prime}\right)=(\lambda+i \epsilon) \delta\left(\lambda-\lambda^{\prime}\right)+\frac{1}{16 \pi^{2} E} \Gamma_{p_{+}, p_{-}}\left(\lambda, \lambda^{\prime}\right) .
$$

In order to analyze (51) we need to compute $\Gamma_{p_{+}, p_{-}}\left(\lambda, \lambda^{\prime}\right)$ for all values of $\lambda, \lambda^{\prime}$. However, we shall use, for the discussion below, the results of the previous section, which are strictly valid only for small values of $\lambda, \lambda^{\prime}$. Therefore the following analysis should be considered heuristic and qualitative.

Equation (51) is reminiscent of a scattering theory problem and is formally solved by

$$
\Psi=\phi-\frac{1}{16 \pi^{2} E} \frac{1}{\lambda \mathbf{1}+i \epsilon} \Gamma_{p_{+}, p_{-}} \Psi
$$

where $\phi(\lambda)=\delta(\lambda)$ is the solution to the free equation of motion. Using (37) and (47), we note that the amplitude $\Gamma_{p_{+}, p_{-}} / E$ is of order $(E / M)(E / p)^{3 / 2}$ or $J^{-5 / 2}(M / p)^{3 / 2}$. Therefore, for large $J$ we can solve (52) in powers of $\Gamma_{p_{+}, p_{-}}$, with the leading "Born" term given by

$$
\Psi(\lambda) \simeq \delta(\lambda)-\frac{1}{16 \pi^{2} E} \frac{1}{\lambda+i \epsilon} \Gamma_{p_{+}, p_{-}}(\lambda, 0) .
$$

In terms of $\Psi(\lambda)$, the dependence of the wave function on the transverse coordinate $y$ is given by

$$
\Psi(y)=2 \pi\left(\frac{2 E}{\left|p_{+}\right|}\right)^{\frac{1}{3}} \int d \lambda \Psi(\lambda) \operatorname{Ai}(-z) .
$$

The normalization has been chosen for later convenience and we recall that

$$
z=\left(2 E p_{+}^{2}\right)^{\frac{1}{3}}\left(y+\frac{2 p_{+} p_{-}-\lambda}{2 E p_{+}^{2}}\right) .
$$

Using the integral representation for the Airy function $\mathrm{Ai}$, we can write $\Psi(y)$ explicitly as

$$
\Psi(y)=\frac{1}{\left|p_{+}\right|} \int d \lambda d k \Psi(\lambda) e^{\frac{i}{2 E p_{+}^{2}}\left[\left(2 E p_{+}^{2} y+2 p_{+} p_{-}-\lambda\right) k-\frac{k^{3}}{3}\right]} .
$$

Let us now use the results of section 5 In particular, the leading contribution to the $\lambda$-dependence of $\Gamma_{p_{+}, p_{-}}$comes from the implicit dependence 
of $p$ on $\lambda$ within the constant $c_{w}$ in equation (50). Expanding the phase to linear order in $\lambda$, and neglecting any other $\lambda$-dependence, the expression for $\Gamma_{p_{+}, p_{-}}(\lambda, 0)$ is given by

$$
\frac{1}{16 \pi^{2} E} \Gamma_{p_{+}, p_{-}}(\lambda, 0) \simeq \frac{\alpha}{2 \pi i} e^{-i \frac{p}{2 E p_{+}^{2}} \lambda}-\frac{\alpha^{\star}}{2 \pi i} e^{i \frac{p}{2 E p_{+}^{2}} \lambda},
$$

where we compute $p=\sqrt{2 p_{+} p_{-}}$and the constant $\alpha=i(8 \pi E)^{-1} \sum_{w \neq 0} c_{w} \Gamma_{w}^{+}$ at $\lambda=0$. The other term in the phase linear in $\lambda$ is $w^{2} \lambda /(E p)$ and is subleading since $p_{+} \ll E / w^{2}$. Using the fact that $\int d \lambda e^{-i \lambda \eta}(\lambda+i \epsilon)=$ $-2 \pi i \theta(\eta)$, we can write the position space wave function as

$$
\Psi(y) \simeq \frac{1}{\left|p_{+}\right|} \int d k\left(1+\alpha \theta(k+p)-\alpha^{\star} \theta(k-p)\right) e^{\frac{i}{2 E p_{+}^{2}}\left[\left(p^{2}+2 E p_{+}^{2} y\right) k-\frac{k^{3}}{3}\right]} .
$$

This integral can be computed using the saddle point approximation for $\left(2 E p_{+}^{2}\right)^{-\frac{2}{3}}\left(p^{2}+2 E p_{+}^{2} y\right) \gg 1$. In particular, this approximation is valid around $y \sim 0$ since $p^{3} \gg E p_{+}^{2}$. For $y \sim 0$ the two saddle points at $k=$ $\pm \sqrt{p^{2}+2 E p_{+}^{2} y}$ are around $\pm p$ and the $\theta$-functions create discontinuities. More precisely, we obtain

$$
\begin{aligned}
& \left(1+\alpha-\alpha^{\star}\right) g_{+}(y)+g_{-}(y), \quad(y>0) \\
& (1+\alpha)\left[g_{+}(y)+g_{-}(y)\right], \quad(y<0)
\end{aligned}
$$

where $g_{ \pm}(y)$ are the left and right moving waves

$$
\begin{aligned}
g_{ \pm}(y) & =\sqrt{\mp 2 \pi i E}\left(p^{2}+2 E p_{+}^{2} y\right)^{-1 / 4} e^{ \pm \frac{i}{3 E p_{+}^{2}}\left(p^{2}+2 E p_{+}^{2} y\right)^{3 / 2}}, \\
& \simeq 2 \pi\left(\frac{2 E}{\left|p_{+}\right|}\right)^{1 / 3} \frac{\mathrm{Ai}(-z) \mp i \operatorname{Bi}(-z)}{2},
\end{aligned}
$$

with $z$ given by (53) for $\lambda=0$. The interaction term $\Gamma_{p_{+}, p_{-}}$creates a discontinuous behavior around $y=0$, consistent with the conjectured dual description in terms of an orientifold plane [13, 11]. Due to the crudeness of the approximations involved, these ideas clearly require a more thorough analysis.

\section{Conclusions}

The propagation of quantum fields in a geometry with closed causal curves is not unitary order by order in the coupling constant. This breakdown of 
unitarity arises from the interactions of external states with on-shell particle loops that wind the closed causal curves. In this paper we showed that, for a orbifold of three dimensional flat space with closed causal curves, unitarity can be restored for specific values of the Newton coupling constant. The results relied on the eikonal approximation to particle scattering, in a kinematic regime where graviton exchange is dominant. Since in the eikonal approximation one is able to resum the perturbative series expansion, this approximation provides a window to new effects in quantum gravity, such as the quantization of the Newton constant from the unitarity requirement. Alternatively, for fixed coupling constant, one can see this condition as quantizing the orbifold geometry, consistently with the quantization of the extremal BTZ black hole angular momentum. Given that a similar condition holds for generic BTZ black holes, the results of this paper may have profound implications for the dynamics of particle states inside black hole horizons.

We have used the off-shell extension of the eikonal amplitude for $2 \rightarrow 2$ scattering in flat space. Although the explicit form of this amplitude is not known, we have only assumed that such extension has the pole structure of the on-shell amplitude, compatibly with the symmetries of the amplitude itself. Clearly, it would be desirable to explicitly compute the off-shell extension of the amplitude and to check if the additional requirement (49) is satisfied. In the usual flat space computation, one needs to deal with IR divergences, which in principle can be eliminated by considering eikonal scattering in $\mathrm{AdS}_{3}$. We think this is an interesting direction of research to pursue, since new connections between quantum gravity in AdS spaces and dual CFT's may be derived.

Based on the particle effective action derived from the orbifold two-point amplitude for off-shell external states, we gave in the final section a heuristic argument showing that particle states are changed precisely at the chronological singularity. This analysis shows that, at finite values of the coupling, particle states are very different from the free particle wave-functions of section 2.2. In fact, free particle states are unstable beyond the chronological singularity. Hence, for zero coupling constant, the condensation of these fields will change the geometry in the pathological region of space-time [32. It is plausible that, at the end-point of this transition, the coupling constant is fixed at some fixed value and the geometry is that of a wall placed at the chronological singularity. This picture is suggested by a sequence of string dualities that maps the orbifold geometry to an orientifold 8-plane 
and the quantization condition (5) to the quantization of RR charge [11. Our computation of the particle effective action, which sees finite coupling constant effects, is also consistent with this picture. 


\section{Acknowledgments}

The authors wish to thank M. Bianchi, M. Ciafaloni, G. 't Hooft, A. Sagnotti, Y. Stanev and G. Veneziano for useful discussions and correspondence.

The authors thank the hospitality of the Fields Institute for Research in Mathematical Studies, where this work was completed. This work was supported in part by INFN, by the MIUR-COFIN contract 2003-023852, by the EU contracts MRTN-CT-2004-503369, MRTN-CT-2004-512194 and MERG-CT-2004-511309, by the INTAS contract 03-51-6346, by the NATO grant PST.CLG.978785 and by the FCT contracts POCTI/FNU/38004/ 2001 and POCTI/FP/FNU/50161/2003. L.C. is supported by the MIUR contract "Rientro dei cervelli" part VII. Centro de Física do Porto is partially funded by FCT through the POCTI programme.

\section{Appendix A}

We focus on the extremal black hole, with $r_{+}=r_{-}$. Writing $\mathrm{AdS}_{3}$ as the surface

$$
-\left(z^{0}\right)^{2}+\left(z^{1}\right)^{2}+\left(z^{2}\right)^{2}-\left(z^{3}\right)^{2}=-\ell^{2}
$$

the BPS black hole is given by $\mathrm{AdS}_{3} / e^{\kappa}$, where

$$
\kappa=\frac{i}{\sqrt{2}}\left(J_{02}+J_{12}\right)+\frac{i}{\sqrt{2}} \frac{\left(2 \pi r_{+}\right)^{2}}{\ell^{2}}\left(J_{02}-J_{12}+J_{03}-J_{13}\right)
$$

with $i J_{\mu \nu}=z_{\mu} \partial_{\nu}-z_{\nu} \partial_{\mu}$ the generators of the $S O(2,2)$ isometry group. Parameterizing $\mathrm{AdS}_{3}$ with coordinates $x^{a}(a=0,1,2)$

$$
\begin{aligned}
& z^{0}+i z^{3}=-i e^{\frac{i x^{0}}{\ell}} \sqrt{\ell^{2}+\left(x^{1}\right)^{2}+\left(x^{2}\right)^{2}}, \\
& z^{1}+i z^{2}=x^{1}+i x^{2},
\end{aligned}
$$

we recover, in the $\ell \rightarrow \infty$ limit, flat Minkowski space $\mathbb{M}^{3}$ with isometry group $I S O(1,2)$. Moreover, the generators $J_{\mu \nu}$ converge to the generators $K_{a}, L_{a b}$ of $I S O(1,2)$, according to

$$
J_{a b} \rightarrow L_{a b}, \quad \frac{1}{\ell} J_{3 a} \rightarrow K_{a} .
$$

In the limit $\ell \rightarrow \infty$, keeping the energy scale

$$
E=\frac{\ell}{\left(2 \pi r_{+}\right)^{2}}
$$


fixed, we arrive at the orbifold $\mathbb{M}^{3} / e^{\kappa}$ with

$$
\kappa=\frac{i}{\sqrt{2}}\left(L_{02}+L_{12}\right)+\frac{i}{\sqrt{2}} E^{-1}\left(K_{0}-K_{1}\right) .
$$

Introducing light-cone coordinates $x^{ \pm}=\left(x^{0} \pm x^{1}\right) / \sqrt{2}$ and $x=x^{2}$, the metric on the covering space is

$$
d s^{2}=-2 d x^{+} d x^{-}+d x^{2},
$$

and $\kappa$ simplifies to

$$
\kappa=i\left(L_{+x}+E^{-1} K_{-}\right)
$$

\section{Appendix B}

We consider equation (48) for different values $w$, with $\sigma=s-u=8 \Lambda p_{-} / w^{2}$ and $2 p=\sqrt{-t}=2 \Lambda /|w|=\sqrt{\sigma \Lambda / 2 p_{-}}$(we consider the case $p_{-}>0$ for concreteness). Introduce, for notational convenience, the phase

$$
\zeta=e^{i \frac{M}{2 E}}
$$

and the constant $c=8 \Lambda p$. Then (48) reads

$$
-\zeta^{w^{2}} F_{+}\left(c / w^{2}\right)+\zeta^{-w^{2}} F_{-}\left(c / w^{2}\right)=\mathcal{A}\left(c / w^{2}\right)
$$

where we denote $\mathcal{A} \equiv \mathcal{A}_{\text {on-shell }}$ for brevity.

We wish to show, under minimal regularity assumptions, that (54) can be satisfied for all values of $w$ only if $\zeta=1$. To this end, we will only need to assume that $F_{ \pm}, \mathcal{A} \in \mathcal{F}$, where $\mathcal{F}$ is a class of continuous realvalued functions of a real variable defined on intervals $(0, a), a>0$, with the following properties:

i) if $f \in \mathcal{F}$, then either $f=0$ or $f(\sigma) \neq 0$ for $\sigma$ sufficiently close to 0 .

ii) if $f, g \in \mathcal{F}$ and $\left\{\sigma_{w}\right\}$ is a sequence of positive reals converging to zero such that $f\left(\sigma_{w}\right) / g\left(\sigma_{w}\right)$ is bounded, then $f(\sigma) / g(\sigma)$ converges for $\sigma \rightarrow 0$.

iii) if $f, g \in \mathcal{F}$, then $f+g \in \mathcal{F}$.

An example of a class of functions satisfying $i$ ), $i i)$ and $i i i)$ is the one of finite sums of terms $x^{a} \log ^{n}(\sigma) \varphi(\sigma)$, where $a$ is a real number, $n$ is an integer 
and $\varphi$ is holomorphic on a neighborhood of the origin in $\mathbb{C}$ and real on the real axis. This example applies in particular to the case relevant for this paper in section 4.4 thanks to general properties of analyticity of scattering amplitudes.

We first assume that $\zeta \neq \pm 1$. As shown in section 4.4, the function $\mathcal{A}$ is nowhere vanishing for $\sigma \neq 0$. Therefore, if $F_{+}=0$, reality of the functions $F_{-}, \mathcal{A}$ and equation (54) imply that $F_{-}$vanishes arbitrarily close to 0 , so that $F_{-}=0$ by $i i$ ). A similar reasoning applies if $F_{-}=0$. Therefore we can assume, by $i$ ), that $F_{ \pm}, \mathcal{A} \neq 0$ in the range $0<\sigma<1 / W^{2}$ for some $W \in \mathbb{N}$. Since $\zeta \neq \pm 1$, there are arbitrarily large values of $w$ such that $\zeta^{w^{2}}$ is not real. We denote with $S \in \mathbb{N}$ the set of these values of $w$ which are greater than $W$. Equating imaginary parts in condition (54) shows that, for $w \in S$, $F_{+}\left(c / w^{2}\right)=-F_{-}\left(c / w^{2}\right)$, and hence (54) reduces to

$$
2 \operatorname{Re}\left(\zeta^{w^{2}}\right) F_{-}\left(c / w^{2}\right)=\mathcal{A}\left(c / w^{2}\right) . \quad(w \in S)
$$

It follows from condition $i i)$ that the ratios $\pm \mathcal{A}(\sigma) / F_{\mp}(\sigma)$ converge for $\sigma \rightarrow 0$ (to the same limit). Now we distinguish two cases:

a) $\zeta$ is a $k$-th root of unity. If $w \pm 1$ is a multiple of $k$, then $\zeta^{w^{2}}=\zeta$ and $w \in S$. Thus (55) implies that

$$
\pm \lim _{\sigma \rightarrow 0} \frac{\mathcal{A}(\sigma)}{F_{\mp}(\sigma)}=2 \operatorname{Re}(\zeta) .
$$

On the other hand, condition (54) for $w$ multiple of $k$ gives

$$
-\lim _{\sigma \rightarrow 0} \frac{F_{-}(\sigma)}{\mathcal{A}(\sigma)}+\lim _{\sigma \rightarrow 0} \frac{F_{+}(\sigma)}{\mathcal{A}(\sigma)}=1
$$

which is incompatible with the above, as $\zeta \neq 1$, unless $\zeta= \pm i$. In this last case, however, (55) shows that $\mathcal{A}\left(1 / w^{2}\right)=0$ for all odd $w$, which contradicts the result of section 4.4 .

b) $\zeta$ is not a root of unity. In this case $\zeta^{w^{2}}$ is never real for $w \neq 0$ and (55) shows that the sequence $\left\{\operatorname{Re}\left(\zeta^{w^{2}}\right)\right\}$ converges to some limit $\cos \theta$. Note, however, that $\zeta^{(w+1)^{2}}=\zeta^{w^{2}} \zeta^{2 w+1}$ and $\zeta^{2 w+1}$ is dense on the unit circle as we vary $w$. Therefore we can choose $w$ large enough so that $\operatorname{Re} \zeta^{w^{2}}$ is arbitrarily close to $\cos \theta$, and so that $\zeta^{2 w+1}$ is arbitrarily close to any chosen number $\xi$ on the unit circle. By choosing $\xi$ different from $e^{ \pm 2 i \theta}$, we can then make $\operatorname{Re} \zeta^{(w+1)^{2}}$ differ from $\cos \theta$ by a finite amount for arbitrarily large values of $w$, therefore showing that the sequence $\left\{\operatorname{Re}\left(\zeta^{w^{2}}\right)\right\}$ does not converge. 
We finally deal with the case $\zeta=-1$. From (54) we deduce that

$$
-F_{+}\left(c / w^{2}\right)+F_{-}\left(c / w^{2}\right)=(-)^{w} \mathcal{A}\left(c / w^{2}\right) .
$$

Since $\mathcal{A}$ has constant sign near 0 , this relation implies that $-F_{+}(\sigma)+F_{-}(\sigma)$ vanishes arbitrarily close to 0 and is non-vanishing. This is a contradiction by $i$ ii).

\section{References}

[1] J. M. Maldacena, The large $N$ limit of superconformal field theories and supergravity, Adv. Theor. Math. Phys. 2 (1998) 231 arXiv:hep-th/9711200.

[2] S. W. Hawking, Breakdown Of Predictability In Gravitational Collapse, Phys. Rev. D 14 (1976) 2460.

[3] M. Banados, C. Teitelboim and J. Zanelli, The Black hole in three-dimensional space-time, Phys. Rev. Lett. 69 (1992) 1849 arXiv:hep-th/9204099.

[4] M. Banados, M. Henneaux, C. Teitelboim and J. Zanelli, Geometry of the (2+1) black hole, Phys. Rev. D 48 (1993) 1506 arXiv:gr-qc/9302012.

[5] A. Strominger, Black hole entropy from near-horizon microstates, JHEP 9802 (1998) 009 arXiv:hep-th/9712251.

[6] J. D. Brown and M. Henneaux, Central Charges In The Canonical Realization Of Asymptotic Symmetries: An Example From ThreeDimensional Gravity, Commun. Math. Phys. 104, 207 (1986).

[7] G. 't Hooft, Graviton Dominance In Ultrahigh-Energy Scattering, Phys. Lett. B 198 (1987) 61.

[8] D. G. Boulware, Quantum field theory in spaces with closed timelike curves, Phys. Rev. D 46 (1992) 4421 arXiv:hep-th/9207054. J. L. Friedman, N. J. Papastamatiou and J. Z. Simon, Failure of unitarity for interacting fields on space-times with closed timelike curves, Phys. Rev. D 46, 4456 (1992). 
[9] M. Simpson and R. Penrose, Internal Instability in a ReissnerNordstrom Black Hole, Int. Journ. Theor. Physics 7 (1973) 183.

[10] S. W. Kim and K. P. Thorne, Do vacuum fluctuations prevent the creation of closed timelike curves?, Phys. Rev. D 43 (1991) 3929.

[11] L. Cornalba and M. S. Costa, Time-dependent orbifolds and string cosmology, Fortsch. Phys. 52 (2004) 145 arXiv:hep-th/0310099.

[12] L. Cornalba and M. S. Costa, A new cosmological scenario in string theory, Phys. Rev. D 66 (2002) 115 arXiv:hep-th/0203031.

[13] L. Cornalba, M. S. Costa and C. Kounnas, A resolution of the cosmological singularity with orientifolds, Nucl. Phys. B 637 (2002) 378 arXiv:hep-th/0204261.

[14] S. W. Hawking, The Chronology protection conjecture, Phys. Rev. D 46 (1992) 603.

[15] G. T. Horowitz and A. R. Steif, Singular string solutions with nonsingular initial data, Phys. Lett. B 258 (1991) 91.

[16] V. Balasubramanian, S. F. Hassan, E. Keski-Vakkuri and A. Naqvi, A space-time orbifold: A toy model for a cosmological singularity, Phys. Rev. D 67 (2003) 026003 arXiv:hep-th/0202187.

[17] J. Simon, The geometry of null rotation identifications, JHEP 0206 (2002) 001 arXiv:hep-th/0203201.

[18] N. A. Nekrasov, Milne universe, tachyons, and quantum group, Surveys High Energ. Phys. 17 (2002) 115 arXiv:hep-th/0203112.

[19] H. Liu, G. Moore and N. Seiberg, Strings in a time-dependent orbifold, JHEP 0206 (2002) 045 arXiv:hep-th/0204168.

[20] S. Elitzur, A. Giveon, D. Kutasov and E. Rabinovici, From big bang to big crunch and beyond, JHEP 0206 (2002) 017 arXiv:hep-th/0204189.

[21] B. Craps, D. Kutasov and G. Rajesh, String propagation in the presence of cosmological singularities, JHEP 0206 (2002) 053 arXiv:hep-th/0205101.

[22] H. Liu, G. Moore and N. Seiberg, Strings in time-dependent orbifolds, JHEP 0210 (2002) 031 arXiv:hep-th/0206182. 
[23] M. Fabinger and J. McGreevy, On smooth time-dependent orbifolds and null singularities, JHEP 0306 (2003) 042 arXiv:hep-th/0206196.

[24] S. Elitzur, A. Giveon and E. Rabinovici, Removing singularities, JHEP 0301 (2003) 017 arXiv:hep-th/0212242.

[25] L. Cornalba and M. S. Costa, On the classical stability of orientifold cosmologies, Class. Quant. Grav. 20 (2003) 3969 arXiv:hep-th/0302137.

[26] R. Biswas, E. Keski-Vakkuri, R. G. Leigh, S. Nowling and E. Sharpe, The taming of closed time-like curves, JHEP 0401 (2004) 064 arXiv:hep-th/0304241.

[27] J. G. Russo, Cosmological string models from Milne spaces and SL(2,Z) orbifold, Mod. Phys. Lett. A 19 (2004) 421 arXiv:hep-th/0305032.

[28] V. E. Hubeny, M. Rangamani and S. F. Ross, Causal inheritance in plane wave quotients, Phys. Rev. D 69 (2004) 024007 arXiv:hep-th/0307257.

[29] B. Pioline and M. Berkooz, Strings in an electric field, and the Milne universe, JCAP 0311 (2003) 007 arXiv:hep-th/0307280.

[30] M. Berkooz, B. Pioline and M. Rozali, Closed strings in Misner space: Cosmological production of winding strings, JCAP 0408 (2004) 004 arXiv:hep-th/0405126.

[31] M. Berkooz, B. Durin, B. Pioline and D. Reichmann, Closed strings in Misner space: Stringy fuzziness with a twist, JCAP 0410 (2004) 002 arXiv:hep-th/0407216.

[32] M. S. Costa, C. A. R. Herdeiro, J. Penedones and N. Sousa, Hagedorn transition and chronology protection in string theory, arXiv:hep-th/0504102.

[33] M. Levy and J. Sucher, Eikonal Approximation In Quantum Field Theory, Phys. Rev. 186, 1656 (1969).

[34] D. Amati, M. Ciafaloni and G. Veneziano, Superstring Collisions At Planckian Energies, Phys. Lett. B 197, 81 (1987).

[35] D. Kabat and M. Ortiz, Eikonal quantum gravity and Planckian scattering, Nucl. Phys. B 388, 570 (1992) arXiv:hep-th/9203082. 
[36] S. Deser and R. Jackiw, Classical And Quantum Scattering On A Cone, Commun. Math. Phys. 118, 495 (1988).

[37] G. 't Hooft, Nonperturbative Two Particle Scattering Amplitudes In (2+1)-Dimensional Quantum Gravity, Commun. Math. Phys. 117, 685 (1988).

[38] S. Deser, J. G. McCarthy and A. R. Steif, UltraPlanck scattering in $D=3$ gravity theories, Nucl. Phys. B 412, 305 (1994) arXiv:hep-th/9307092. 